NOTICE: This is the author's version of a work that was accepted for publication in Journal of Sound and Vibration. Changes resulting from the publishing process, such as peer review, editing, corrections, structural formatting, and other quality control mechanisms may not be reflected in this document. Changes may have been made to this work since it was submitted for publication. A definitive version was subsequently published in Journal of Sound and Vibration, Vol. 332, Issue (26). (2013). doi: 10.1016/j.jsv.2013.08.012 


\title{
AERO-/HYDRO-ELASTIC STABILITY OF FLEXIBLE PANELS: PREDICTION AND CONTROL USING LOCALISED SPRING SUPPORT
}

\author{
B.H. Tan, A.D. Lucey* \& R.M. Howell \\ Fluid Dynamics Research Group, Curtin University of Technology, \\ P.O. Box U1987, Perth 6845, Australia.
}

*Corresponding author: Tel.: +61-8-9266-7048, Fax.: +61-8-9266-2681, E-mail: A.Lucey@curtin.edu.au

\begin{abstract}
We study the effect of adding localised stiffness, via a spring support, on the stability of flexible panels subjected to axial uniform incompressible flow. Applications are considered that range from the hydro-elasticity of hull panels of high-speed ships to the aero-elasticity of glass panels in the curtain walls of high-rise building in very strong winds. A two-dimensional linear analysis is conducted using a hybrid of theoretical and computational methods that calculates the system eigen-states but can also be used to capture the transient behaviour that precedes these. We show that localised stiffening is a very effective means to increase the divergence-onset flow speed in both hydro- and aero-elastic applications. It is most effective when located at the mid-chord of the panel and there exists an optimum value of added stiffness beyond which further increases to the divergence-onset flow speed do not occur. For aero-elastic applications, localised stiffening can be used to replace the more destructive flutter instability that follows divergence at higher flow speeds by an extended range of divergence. The difference in eigen-solution morphology between aero- and hydro-elastic applications is highlighted, showing that for the former coalescence of two non-oscillatory divergence modes is the mechanism for flutter onset. This variation in solution morphology is mapped out in terms of a non-dimensional mass ratio. Finally, we present a short discussion of the applicability of the stabilisation strategy in a full three-dimensional system.
\end{abstract}

Keywords: Fluid-structure Interaction, Aero-/Hydro-elasticity, Flexible Plate, Divergence/Flutter Instability, Structural Inhomogeneity, Eigen-analysis, Boundary-/Initial-value Problem 


\section{INTRODUCTION}

This paper addresses and extends the classical fluid-structure interaction (FSI) problem wherein a flexible plate is destabilized by the action of a fluid flow parallel to the undisturbed panel. The modern capabilities of high-speed ships with cruise speeds in the range to 38-45 knots (19.5-23.1 $\mathrm{m} / \mathrm{s})$ - and up to 60 knots $(30.1 \mathrm{~m} / \mathrm{s})$ when powered by gas-turbine engines - means that hydroelastic instability increasingly needs to be accounted for in the design of hull panels. Recent architectural designs have seen the introduction of curtain walls comprising glass or perspex panels as an outer skin on high-rise buildings for a combination of aesthetic and passive temperature-control reasons. In addition to normal-loading effects, these may be susceptible to aeroelastic instability in storm or hurricane-force winds aligned with the main axis of the panel. In this paper we present an analytical study of panel stability into which localised stiffening is added and used to control aero/hydro-elastic instability in the above and other applications of the basic configuration.

The high Reynolds-number regime typical of the types of engineering applications cited above makes the neglect of viscous effects on the flow a good approximation. Accordingly, potential flow is most often assumed as is the case in this study. Given the importance and ubiquity of applications, this FSI system has generated a rich literature in which, most commonly, a Galerkin method is used to predict the system response with a particular focus on the parameters for which it becomes unstable. Thus, for example, [1-5], show that as the flow speed is increased for a given flexible plate, the panel first loses its stability to divergence. This buckling type of instability occurs because the fluid forces generated by a deformation exceed the restorative structural forces of that deformation. For a simple flexible plate held at both its ends, the fundamental mode is the critical mode for divergence. If the flow speed is increased further, divergence is replaced by modal-coalescence flutter that is best characterized as a Kelvin-Helmholtz type of resonance.

In parallel to these types of study, flexible compliant walls of infinite extent comprising more than one structural component (e.g. a spring-backed flexible plate) have been studied, e.g. [1,6,7] using an analytical approach wherein all system perturbations take a travelling-wave form, for example $\exp [\mathrm{i}(\alpha x-\omega t)]$ wherein $\alpha$ and $\omega$ are respectively the perturbation wavenumber and angular 
frequency. The omission of end effects - that may be considered to be inhomogeneities in such modelling - is broadly acceptable provided that the length of the panel in any application is much longer than the wavelength of the critical modes being studied. However, under such conditions the travelling-wave analysis requires that some structural damping is present for the realisation of divergence instability although its predictions of divergence-onset flow speed agree with those of the Galerkin approach. This discrepancy was addressed in [8] wherein the role of end conditions, even for very long flexible walls was explained. More recently the rigorous analysis of [9] constructed a travelling-wave model that incorporated the fixed wall ends through a Weiner-Hopf technique and thereby reconciled the differences in findings between the two types of modelling.

Clearly, the aforementioned boundary-value studies predict the long-time response of the system after transients from some form of initial excitation have either been attenuated or convected away. The finite-time response can be of equal importance in that it links the original source and characteristics of an initial deformation to the long-time response through a process of response evolution. The ability to model the finite-time, or receptivity, problem may lead to engineering strategies that interrupt or modify this evolution and thereby prevent or postpone panel instability. Studies of system response to a source of initial or continuing localized excitation have been presented. For example, [10] and [11] respectively used initial impulse and oscillatory line excitation for the present system, while [12] tackled the closely related shell problem with oscillatory line excitation. Using a different analytical approach, [13] showed that absolute instability - that aligns with divergence - could exist in the system if structural damping were included. These analyses assumed an infinitely long flexible panel and focused on the long-time response. Nevertheless, they showed that the system could support a remarkable range of FSI wave types. Using numerical simulation, [14] showed that the effects of finiteness and transients led to globally unstable responses unseen in the analyses of infinitely long elastic panels.

In the present work, we use the hybrid of theoretical and computational modelling presented in [15] that casts the FSI system equation in state-space form after solving the coupled fluid and structure equations using boundary-element and finite-difference methods respectively. Like the 
purely analytical models discussed above, this approach is used to compute the system eigenmodes while its numerical-simulation aspects readily accommodate inhomogeneity in the base system. Thus we can evaluate the effect of an added localised spring support on the system eigenmodes with a particular focus on instability-onset flow speeds. We also extend the modelling of [15] in order to solve the initial-value problem and thereby simulate the transient response of finite flexible panels showing how its evolution from a source of initial excitation evolves into the infinite-time eigenmodes predicted by the boundary-value approach.

The paper is laid out as follows: We first extend the FSI system model of [15] to permit the inclusion of impulse line excitation and a supporting spring foundation that may either be uniform or comprise a discrete spring at a point along the flexible plate. We then present three sets of results that illustrate the system dynamics covering a range of applications. The first concerns a homogeneous Kramer-type compliant wall [7] comprising a flexible plate with a uniformly distributed spring foundation. In part, we use this case to validate the present modelling and its implementation. The second set of results addresses the classical case of a simple metal flexible panel subjected to water flow for which we show how the addition of a spring support can be used to modify hydroelastic instability onset. This case typifies the vast majority of incompressible flow studies for which the fluid-to-solid ratio is $O(1)$. In the third set of results we consider airflow over a glass or aluminium panel for which the fluid-to-solid ratio is $O\left(10^{-3}\right)$, giving a system that has not hitherto been fully explored, presumably due to a lack of recognised applications until the emergence of curtain walls as an architectural feature. We show that this regime possesses some very different dynamics from the classical hydro-elastic case. We therefore map out the parameter space over which the differences occur as well as showing how adding a spring support can modify both divergence onset and the flutter characteristics in air-over-glass aero-elastic applications. Finally we unify our findings in the conclusions and explain how the present two-dimensional strategy for controlling aero-/hydro-elastic instability of panels can be carried across to real three-dimensional applications. 


\section{METHODS}

We first summarise the well-known governing equations for the fluid-structure systems depicted in Fig. 1. We then outline the hybrid theoretical-computational approach that permits either an eigen-analysis to be conducted for the long-time boundary-value problem or the time-evolution of disturbances to be constructed for the initial-value problem. Finally, we describe the nondimensional framework adopted for the presentation of the results in this paper.

\subsection{Governing equations}

The small-amplitude behaviour of a thin plate, supported by either a uniformly distributed spring foundation with coefficient $K \neq 0$, and an added spring support with coefficient $k \neq 0$ localised at $x=x_{k}$, in the presence of a fluid flow and subjected to a localised initial pressure pulse of magnitude $p_{e}$ at $x=x_{p}$ is governed by

$$
\rho_{m} h \ddot{\eta}+d \dot{\eta}+B \eta_{, x x x x}+K \eta+k \delta\left(x-x_{k}\right) \eta=-p(x, 0, t)+p_{e} \delta\left(x-x_{p}\right) \delta(t)
$$

where $\eta(x, t), \rho_{m}, h, d$ and $B$ are, respectively, the plate's deflection, density, thickness, (dashpottype) damping coefficient and flexural rigidity (evaluated using $B=E h^{3} /\left[12\left(1-\nu^{2}\right)\right]$ where $\nu$ is the Poisson ratio of the plate material), $\delta$ is the Dirac delta function, and $p(x, 0, t)$ is the fluid-pressure perturbation that acts to deform the plate, noting that the mean transmural pressure is such that the plate's mean position lies in the plane $y=0$. The flexible plate of length $L$ is hinged at its leading and trailing edges giving $\eta(0, t)=\eta_{, x x}(0, t)=\eta(L, t)=\eta_{, x x}(L, t)=0$. We use overdot and suffix notations for temporal and spatial derivatives respectively.

The flow is assumed to be incompressible and irrotational, allowing the introduction of a velocity perturbation potential $\phi(x, y, t)$ that satisfies Laplace's equation

$$
\phi_{, x x}+\phi_{, y y}=0
$$

with the condition that $\phi \rightarrow 0$ as $y \rightarrow \infty$. The unsteady fluid pressure is determined using the linearised unsteady Bernoulli equation

$$
p=-\rho_{f} \dot{\phi}-\rho_{f} U_{\infty} \phi_{, x}
$$


where $\rho_{f}$ and $U_{\infty}$ are, respectively, the fluid density and flow speed. The plate and fluid motions are coupled through the kinematic boundary condition

$$
\phi_{, y}=\dot{\eta}+U_{\infty} \eta_{, x}
$$

which in the linearised system is enforced at $y=0$.

\subsection{Solution methods}

The governing equations are solved by combining a boundary-element method for the flow field, as developed in $[16,17]$ for problems in FSI, with a finite-difference method for the wall motion. This reduces the two-dimensional field problem to a one-dimensional line problem at the interface of the fluid and structural components of the system. The resulting system equation, couched in the interfacial variable $\eta(x, t)$ and its differentials, is then cast in state-space form following the approach developed in [15]. In the present work, we make the straightforward extension of these methods to (i) incorporate spatial inhomogeneity in the form of an isolated spring support, and (ii) model the initial-value problem. Accordingly, we provide herein only an outline and direct readers to the papers cited above for details of the contributing elements of the solution procedures.

The panel is discretised into $N$ collocation points at which its mass is lumped and which provide the basis for writing Eqn. (1) in the finite-difference form

$$
\rho_{m} h[I]\{\ddot{\eta}\}+d[I]\{\dot{\eta}\}+\left(B\left[D_{4}\right]+K[I]+\left[0_{k}\right]\right)\{\eta\}=-\{p\}+\delta(t)\left\{0_{p}\right\}
$$

where $[I]$ is the identity matrix and $\left[D_{4}\right]$ is the penta-diagonal fourth-order differentiation matrix operator. $\left[0_{k}\right]$ is a null matrix except for its element $(m, m)$ which has the value $k$ where $m=$ $\operatorname{int}\left[\left(x_{k} / L\right) N\right]$ is the collocation point closest to the location at which the spring has been added. Although we add only one isolated spring support in this study, clearly any number of such springs

could be modelled using our approach. On the right-hand side of Eqn. (5), the pressure pulse appears as the value $p_{e}$ at location $n=\operatorname{int}\left[\left(x_{p} / L\right) N\right]$ in the otherwise null vector $\left\{0_{p}\right\}$.

The pressure perturbation due the motion of the wall is obtained by constructing a solution to the Laplace equation (2) using a source-sink singularity distribution, discretising this using a set of 
boundary-elements based upon the $N$ collocation points, enforcing the no-flux condition, Eqn. (4), at the $N$ control points of the boundary elements to determine the required singularity strengths, and finally using the linearised Bernoulli equation (3) along a surface streamline; this gives the result

$$
-\{p\}=2 \rho[\Phi]\left[D^{+}\right]\{\ddot{\eta}\}+2 \rho U_{\infty}\left([\Phi]\left[D_{1}\right]+[T]\left[D^{+}\right]\right)\{\dot{\eta}\}+2 \rho U_{\infty}^{2}[T]\left[D_{1}\right]\{\eta\}
$$

where $[\Phi]$ and $[T]$ are respectively the matrices of perturbation-potential and tangential-velocity influence coefficients, $\left[D_{1}\right]$ is the first-order differentiation finite-difference matrix operator and $\left[D^{+}\right]$ is a matrix operator for the interfacial vertical speed; expressions for the influence coefficients are listed in [17].

Substitution of Eqn. (6) into Eqn. (5) then yields the fluid-structure system equation

$$
[A]\{\ddot{\eta}\}+[B]\{\dot{\eta}\}+[C]\{\eta\}=-\delta(t)\left\{0_{p}\right\}
$$

where

$$
\begin{aligned}
& {[A]=-\rho_{m} h[I]+2 \rho[\Phi]\left[D^{+}\right]} \\
& {[B]=-d[I]+2 \rho U_{\infty}\left([\Phi]\left[D_{1}\right]+[T]\left[D^{+}\right]\right),} \\
& {[C]=-B\left[D_{4}\right]-K[I]-\left[0_{k}\right]+2 \rho U_{\infty}^{2}[T]\left[D_{1}\right] .}
\end{aligned}
$$

Introducing the $2 \times N$ vector of state variables $\{x\}^{T}=\{\{\eta\},\{\dot{\eta}\}\}^{T}$, allows the system equation (7) to be re-written as

$$
\{\dot{x}\}=[H]\{x\}+\delta(t)\{G\}
$$

in which

$$
[H]=\left[\begin{array}{cc}
0 & I \\
-[A]^{-1}[C] & -[A]^{-1}[B]
\end{array}\right] \quad \text { and } \quad\{G\}=\left\{\begin{array}{c}
0 \\
{[A]^{-1}\left\{0_{p}\right\}}
\end{array}\right\}
$$

To determine the long-time response of the system we omit the initial excitation in Eqn. (9), assume single-frequency response proportional to $\exp (\omega t)$, and then solve the eigenvalue problem to determine the $2 N$ values of $\omega=\omega_{R}+\mathrm{i} \omega_{I}$. The real part, $\omega_{R}$, gives the amplification/decay while the imaginary part $\omega_{I}$ is the angular frequency of the eigenmode. 
To solve the initial-value problem we apply a zero-order hold on the input, $\{G\}$, of Eqn. (9) to digitize the continuous system $(H, G)$. Time scaling is used to transform state-space solution into a sampled system that is then solved using MATLAB functions to determine the transient response of the system.

\subsection{Non-dimensional framework}

For simple panels with length $L$ characterising the wavelength of typical flow-induced deformations, it is common (e.g. $[3,18,4,15])$ to non-dimensionalise the FSI system using this lengthscale along with timescale defined as $L / U_{\infty}$. This yields the fluid-to-structure stiffness ratio $\Lambda=\rho_{f} U_{\infty}^{2} L^{3} / B$ as a control parameter for a system with a given (solid-to-fluid) mass ratio, $\mu=\rho_{m} h /\left(\rho_{f} L\right)$. In the present work that features spring supports, $L$ does not necessarily represent the wavelength of critical modes in the destabilisation of a panel through divergence and flutter. Accordingly, we non-dimensionalise using a reference length-scale, $l_{\text {ref }}$ and timescale $t_{\text {ref }}$ defined by

$$
l_{\mathrm{ref}}=\frac{\rho_{m} h}{\rho_{f}} \quad \text { and } \quad t_{\mathrm{ref}}=\frac{\left(\rho_{m} h\right)^{5 / 2}}{\rho_{f}^{2} B^{\frac{1}{2}}}
$$

thereby following the scheme used in $[10,11,19]$ that reduces an infinitely-long, unsupported elasticplate problem to a system of equations with just one non-dimensional control parameter, namely the flow speed.

Using the forms in Eqn. (11a,b) we define the non-dimensional terms

$$
x^{\prime}, \eta^{\prime}=\frac{x, \eta}{l_{\mathrm{ref}}}, t^{\prime}=\frac{t}{t_{\mathrm{ref}}}, d^{\prime}=d\left[\frac{\left(\rho_{m} h\right)^{\frac{3}{2}}}{\rho_{f}^{2} B^{\frac{1}{2}}}\right], K^{\prime}, k^{\prime}=K, k\left[\frac{\left(\rho_{m} h\right)^{4}}{\rho_{f}^{4} B}\right], p^{\prime}=p\left[\frac{\left(\rho_{m} h\right)^{3}}{\rho_{f}^{3} B}\right],(12 a-e)
$$

that give the non-dimensional form of the system equation as

$$
\ddot{\eta}^{\prime}+d^{\prime} \dot{\eta}^{\prime}+\eta_{, x^{\prime} x^{\prime} x^{\prime} x^{\prime}}^{\prime}+K^{\prime} \eta^{\prime}+k^{\prime} \delta\left(x^{\prime}-x_{k}^{\prime}\right) \eta=-p^{\prime}+p_{e}^{\prime} \delta\left(x^{\prime}-x_{p}^{\prime}\right) \delta\left(t^{\prime}\right)
$$

with panel length, often termed the mass ratio, and applied flow speed taking the non-dimensional forms

$$
L^{\prime}=\frac{\rho_{f} L}{\rho_{m} h} \quad \text { and } \quad U^{\prime}=U\left[\frac{\left(\rho_{m} h\right)^{\frac{3}{2}}}{\rho_{f} B^{\frac{1}{2}}}\right]
$$


This approach allows us to vary independently the key parameters of panel length and applied flow speed in the non-dimensional results that follow. The relationship between the often-used stiffness ratio, $\Lambda=\rho_{f} U_{\infty}^{2} L^{3} / B$, and the two system variables used in this paper is

$$
\Lambda=\left(U^{\prime}\right)^{2}\left(L^{\prime}\right)^{3}
$$

Furthermore, for a panel with given geometric properties, $L$ and $h$, variations to $L^{\prime}$ can be interpreted as changing the fluid-to-solid density ratio, $\rho_{f} / \rho_{m}$, thereby highlighting the differences between the aero- and hydro-elastic behaviour of a given panel.

\section{RESULTS}

We present results for three related engineering systems. Although our results are presented in a non-dimensional form that spans the physical parameter space, each system aligns with a distinct engineering application, these being water flow over a rubber-like compliant wall (Section 3.1) with drag-reduction capabilities, water flow over a simple metal panel (Section 3.2) typical of that used for the hulls of high-speed ships, and air flow over simple glass panels (Section 3.3) typical of curtain walls in modern high-rise buildings. For the last two applications we close with a dimensional demonstration of how instability can be controlled by using a localised spring support for the applications cited. We do not present a separate validation of the present method because that has already been done for the base method in [15] while the validation of our extensions to the method are embedded in the new results upon which we focus.

\subsection{Flexible panel with uniformly-distributed spring foundation}

We consider the spring-backed flexible-plate configuration of Fig. 1a, incorporating structural damping, that approximates a compliant coating of the type investigated in, for example, [7, 15, $17,20]$. The physical properties of this wall have $h=0.01 \mathrm{~m}, \rho_{m}=852 \mathrm{~kg} / \mathrm{m}^{3}, B=4.44 \times 10^{-2}$ $\mathrm{Nm}$ (having used $E=4 \times 10^{5} \mathrm{~N} / \mathrm{m}^{2}$ and $\nu=0.5$ ),$d=2.0 \times 10^{4} \mathrm{Ns} / \mathrm{m}^{3}$ and $K=3.68 \times 10^{7}$ $\mathrm{N} / \mathrm{m}^{3}$; the length, $L$, of the flexible panel is $0.6 \mathrm{~m}$ and the fluid is water with density $\rho=10^{3}$ $\mathrm{kg} / \mathrm{m}^{3}$. These data yield the non-dimensional parameter values $d^{\prime}=2.36, K^{\prime}=4.41$ and $k^{\prime}=0$ in the governing equation (13) and $L^{\prime}=70.4$ in Eqn. (14a). 
With $p_{e}^{\prime}=0$ in Eqn. (13), the infinite-time behaviour of the system is represented by the variation of its eigenvalues with non-dimensional flow speed in Figs. $2 \mathrm{a}$ and $2 \mathrm{~b}$ that respectively chart the real (positive denoting amplification and negative decay) and imaginary (oscillatory) parts of the eigenvalues non-dimensionalised using the reference time defined in Eqn. (11b). Only the 40 eigenvalues having the lowest oscillatory frequencies are plotted although all 1600 were calculated having discretised the wall into $N=800$ elements. The onset of divergence instability is seen to occur at $U^{\prime}=2.326$ where the locus of the mode that yields this critical speed first crosses into the amplifying quadrant of Fig. 2a and its oscillatory frequency becomes zero in Fig. 2b; the latter feature has led to this instability often been termed static divergence in the very early studies of compliant walls such as $[6,21]$.

The envelope formed by the closely spaced discrete modes seen in Fig. 2 suggests that the system can be modelled by a continuous spectrum of modes that is assumed in the normal-mode decomposition used to analyse walls of infinite extent. Using this approach, also referred to as a travelling-wave analysis wherein all system disturbances are proportional to exp $[\mathrm{i}(\alpha x-\omega t)]$, [7] derived analytical expressions for the critical, or lowest, flow speed for divergence-onset and determined the wavelength $(\lambda=2 \pi / \alpha)$ of the critical mode that has $\omega=0$ at divergence onset; these were respectively given by

$$
U_{D}=2\left(\frac{B K^{3}}{27 \rho_{f}}\right)^{\frac{1}{8}} \quad \text { and } \quad \lambda_{D}=2 \pi\left(\frac{3 B}{K}\right)^{\frac{1}{4}}
$$

Using the present wall properties Eqn. (16a) gives the critical flow speed $U_{D}=19.51 \mathrm{~m} / \mathrm{s}$ that, when non-dimensionalised yields $U_{D}^{\prime}=2.303$. This is approximately $1 \%$ lower than our prediction of 2.326 from Fig. 2. Exact agreement could only be expected in the limit of infinite plate length and the present prediction for a plate of finite length is expected to be higher, as found, because of the structural restraints at its leading and trailing edges. This correlation supports the integrity of our approach and its implementation.

Using our transient analysis, we now solve an initial-value problem to map out the system behaviour that would lead to the establishment of the infinite-time behaviour predicted by Fig. 
2. This type of analysis also serves to distinguish valid modes from physically unachievable, or spurious, system states that can be predicted in an eigen-solution. A line impulse is applied at the centre of the undisturbed compliant-wall panel to initiate motion. The time sequence of profiles in Fig. 3a shows wall deformations at $U^{\prime}=2.320$, incrementally below the predicted divergence-onset speed, so as to simulate the marginally stable state that exists just before divergence onset. At early times flow-modified flexural waves propagate outwards from the impulse while at later times the wall settles into a nearly uniform mode across the entire wall in which fit approximately 12 wavelengths. This mode would be almost identical to the critical mode of divergence onset at a marginally higher flow speed. However, in the present simulation, amplitude decay occurs through the action of the structural damping. The wall would therefore return to its undisturbed state in the long-time limit when all of the energy initially input by the excitation has been dissipated. Again, good agreement is found with the travelling-wave based predictions of [7]; for the present physical data, Eqn. (16b) predicts a critical wavelength of $0.049 \mathrm{~m}$ that would yield close to 12 disturbance wavelengths on the present finite wall of length $0.6 \mathrm{~m}$. A much higher flow speed, $U^{\prime}=3.867$, than that of divergence onset is used to generate Fig. 3(b) to illustrate unstable behaviour. Rapid amplitude growth of system disturbances is seen to propagate in both upstream and downstream directions from the point of initial excitation. This is characteristic of absolute instability as defined in [22]. For the present fluid-structure system it is discussed and demonstrated theoretically and numerically in [23] and for a cylindrical shell in [12] that has a similar solution morphology to that of a spring-backed flexible plate. In [12] and [23] it is shown that the addition of a further structural component - a spring backing to a flexible plate or hoop stress in a cylindrical shell - causes a very significant increase to Crighton \& Oswell's [11] flow speed of $U^{\prime}=0.074$ for the onset of absolute instability. This is reflected by the critical flow speeds, $U_{D}^{\prime}=2.303$ and 2.326 for infinitely long and the present finite walls respectively, of instability-onset determined herein; we return to this point in Section 3.2 where the flexible wall is similar to that studied by Crighton \& Oswell. The importance of absolute instability owes itself to the fact that it can spread to all locations of the compliant wall irrespective of the location of the initial, or a continuing ([14]), excitation. 


\subsection{Flexible panel with a single-spring support: hydroelastic applications}

The investigation of this paper now focuses on the hydro-elasticity of a simple elastic panel held at its ends. The purpose here is to show that the addition of an isolated spring support between the two ends, as shown in Fig. 1b, can control the onset and form of instability. The dimensional properties used correspond to those of an aluminium panel with $h=0.0025 \mathrm{~m}, \rho_{m}=2600 \mathrm{~kg} / \mathrm{m}^{3}$, and $B=76.62 \mathrm{Nm}$ (having used $E=5.52 \times 10^{10} \mathrm{~N} / \mathrm{m}^{2}$ and $\nu=0.25$ ). The spring-stiffness coefficient is $k_{s}=\int k \delta\left(x-x_{k}\right) \mathrm{d} x$ and initially explored in multiples, $n$, of $k_{+}=6.0 \times 10^{3} \mathrm{~N} / \mathrm{m}^{2}$, hence $k_{s}=n k_{+}$. The length, $L$, of panel is $0.6 \mathrm{~m}$ (although we close this sub-section with results that show the dependence of divergence instability upon $L / h)$ and the fluid is water with density $\rho=10^{3} \mathrm{~kg} / \mathrm{m}^{3}$. The non-dimensional parameter values in the governing equation (13) are therefore $d^{\prime}=0, K^{\prime}=0, k^{\prime}$ selected so that $k_{+}^{\prime}=2.15 \times 10^{-5}$, and with $L^{\prime}=92.3$ in Eqn. (14a).

Figures $4 \mathrm{a}$ and $4 \mathrm{~b}$ respectively shows the variation of the real and imaginary parts of the two lowest-frequency system eigenmodes with flow speed; however, all $2 N=400$ eigenmodes are included in the solution of the FSI system. Three sets of data, $k_{s}^{\prime}=n k_{+}^{\prime}$ with $n=0,6$ and 15 for a spring support added at the panel mid point $x / L=0.5$, are presented, the first giving the result for the standard panel studied many times before, for example $[2,3,18,24]$ and the homogeneous results of [15]. At low flow speeds, the fluid-loaded panel undergoes neutrally stable oscillations. Figure $4 \mathrm{~b}$ shows that the oscillation frequency in the first mode is increased by the inclusion of the spring but that the second mode is almost unaffected because the spring has been placed at the nodal point of this mode. As the flow speed is increased a bifurcation is seen to occur at $U^{\prime}=0.00714$ for the homogeneous case. This is the onset of divergence instability at which the flow speed is sufficiently high that the hydrodynamic stiffness - the last term on the right-hand side of Eqn. (6) - exactly balances the panel's structural restorative forces. Combining the present critical flow speed $U^{\prime}=0.00714$ with the present length of the panel, $L^{\prime}=92.31$, through Eqn. (15) gives the non-dimensional stiffness ratio $\Lambda=40.1$ for divergence-onset. This is in excellent agreement with the predictions of the aforementioned studies of homogeneous plates. Beyond this threshold a positive real part of the eigenvalue appears in Fig. 4a that commences the divergence 
loop of instability. As the added-spring constant is increased the divergence-onset flow speed, for a panel of given flexural rigidity, is seen to increase to 0.0123 for $6 k_{+}^{\prime}$ and 0.0174 for $15 k_{+}^{\prime}$. At higher flow speeds there is a recovery from divergence but soon after modal-coalescence (of the first two modes) flutter occurs at a flow speed of 0.0199 for the standard case $\left(0 k_{+}^{\prime}\right)$, at 0.0203 for $6 k_{+}^{\prime}$ and 0.0210 for $15 k_{+}^{\prime}$. The very significant increase in divergence-onset flow speed as $k_{s}^{\prime}$ is increased, together with the lesser postponement of flutter, suggest a simple strategy for extending the envelope of stable operation of fluid-loaded panels in engineering applications.

We now show how the added spring contributes to the postponement of divergence in terms of energy budgets. The dimensional wall energy comprises three parts, namely plate strain energy $E_{S}$, plate kinetic energy $E_{K}$ and the stored energy of the added spring $E_{S P}$, respectively defined as

$$
E_{S}=\frac{1}{2} B \int_{0}^{L} \eta_{, x x}^{2} \mathrm{~d} x, \quad E_{K}=\frac{1}{2} \rho_{m} h \int_{0}^{L} \eta_{, t}^{2} \mathrm{~d} x, \quad \text { and } \quad E_{S P}=\left.\frac{1}{2} k_{s} \eta^{2}\right|_{x=x_{p}} . \quad(17 a, b, c)
$$

[14] also introduced a term called the virtual work done by the hydrodynamic stiffness component, $p_{s}$ - the part of of the pressure that is dependent upon the interfacial displacement (the final term on the right-hand side of Eqn (6)) - in the establishment of a wall deformation. This is defined as

$$
E_{V W}=-\frac{1}{2} \int_{0}^{L} \eta p_{s} \mathrm{~d} x
$$

Each of the terms in Eqns. (17) and (18) is non-dimensionalised through multiplication by $\left(\rho_{m} h\right) /\left(\rho_{f} B\right)$, consistent with the scheme outlined in Section 2.3. The eigenmodes, $\eta(x, t)$, corresponding to the eigenvalues of Fig. 2 are normalized and thus the non-dimensional energy terms are scaled for an amplitude of unity. It was shown in [14] that divergence onset can be defined as the flow speed for which $E_{V W}$ exactly balances the mechanical energy of the wall. To show how this occurs, both without and with an added spring, we present Fig. 5. Figure 5a corresponds to the standard result in Fig. 4 and is a time-stepping numerical evaluation of the Mode-1 energy terms for a flow speed marginally lower than that of divergence onset at $U^{\prime}=0.00710$. Because the flow speed is so close to that of divergence onset, where structural and hydrodynamic forces nearly balance each other, 
the wall acceleration and velocity are very small, hence the insignificant values of $E_{K}$ in the plot and the slow oscillation of the wall. The key feature is that $E_{V W}$ is almost exactly balanced with the plate's strain energy, $E_{S}$. Figure $5 \mathrm{~b}$ is the equivalent result with a spring added at the panel mid-point and corresponds to the result of Fig. 4 for $k_{s}^{\prime}=6 k_{+}^{\prime}$. The evaluation is conducted at $U^{\prime}=0.0120$ slightly lower than that of divergence onset. It is now seen that both the strain energy of the plate and the spring energy contribute to the total value of mechanical energy that balances $E_{V W}$ and the proportions in which they do so. In this particular case, it is evident that most of the wall's restorative force is provided by the added spring in the stabilisation strategy.

To illustrate the effect of the added spring support on the key fluid-structure modes that would be most evident in the system response, we present Figs. 6, 7 and 8 obtained using $k_{s}^{\prime}=6 k_{+}^{\prime}$. Figure 6 shows the neutrally stable oscillations at $U^{\prime}=0.00378$ as a set of panel profiles over one cycle corresponding to the eigenvalues of Modes 1,2 and 3 calculated using the analysis that generated Fig. 4. The thick lines show the initial and final positions of the panel. While superficially Mode 1 appears similar to the fundamental mode for a standard panel (see [15] for comparisons), the effect of the spring added at the mid-point has clearly introduced the next higher harmonic. Similarly Mode 2 can be seen to contain an element of the fourth harmonic albeit at a lower intensity. This progression continues with Mode 3 for which the higher sixth higher harmonic is perceptible upon very close inspection . Further results (not presented here) for example at $k_{s}^{\prime}=15 k_{+}^{\prime}$ serve to increase the contribution of these 'wavelength-doubling' harmonics; this phenomenon could be expected given that in the limit of infinite added-spring stiffness the flexible panel effectively becomes two separate panels. Figure 7 , obtained at $U^{\prime}=0.0151$ in the divergence loop of Fig. 4 ( $6 k_{+}^{\prime}$ result), shows the amplifying and accompanying decaying modes of the instability over a sequence of equal time-steps. Growth is seen to occur as a quasi-downstreamtravelling wave while the attenuating wave, that would not be evident in the physical system (demonstrated in the initial-value problem presented below) is a quasi-upstream-travelling wave. Further results show that increasing $k_{s}^{\prime}$ from the value used in Fig. 7a causes greater downstream distortion of the wave so as to increase the ratio of the peak amplitude to the deflection of the panel 
mid-point where the spring has been located. Figure 8 shows the amplifying mode of the complexconjugate pair at $U^{\prime}=0.0227$ in the regime of flow speeds of Fig. 4 for which Modes 1 and 2 have coalesced to give a powerful flutter instability. Figure 8a shows the actual sequence of deformations while in Fig. 8b amplitude growth has been (artificially) suppressed so as to give a clearer picture of the mode shape. Animations show that the flutter effectively occurs as a travelling wave that 'sloshes' between the leading- and trailing-edge constraints as demonstrated theoretically by [4]. The mode shape principally comprises a natural Mode 2 shape whereas the standard unsupported case features significant Mode 3 content ([15]). This is because the presence of the added spring predisposes the panel to a reduction of the mid-point deflection for which Mode 2 has a lower energy intensity in the combined plate-plus-spring system.

The foregoing results of this sub-section predict the infinite-time response of the system - the boundary-value problem. We now investigate how such responses might come into being from a source of localised initial excitation in finite time by a time-stepping solution of the initial-value problem. The merit of such an enquiry is that understanding the mechanism through which panel instability comes into being might lead to the development of intervention strategies by which it can be forestalled. The undeformed plate is subjected to a line impulse at $t^{\prime}=0$ modelled as non-zero $p_{e}^{\prime}$ applied at the plate's mid-point in Eqn. (13) for the same case, $k_{s}^{\prime}=6 k_{+}^{\prime}$ as Figs. 7 and 8. Figure 9 shows the development of divergence instability at a flow speed $U^{\prime}=0.0151$ for the case modelled in Fig. 7. Immediately after the applied excitation Fig. 9a shows that the plate response is characterised by very high frequency low-wavelength waves that travel outwards from the point of initial excitation. These are essentially flexural waves of the plate - the structural-force intensity far outweighs the pressure loading - possessing a range of frequencies because the input impulse is a wide-spectrum excitation. These waves are neutrally stable and serve to propagate disturbance energy upstream and downstream of the original input source. The evident amplitude growth over this sequence of time-steps might suggest instability. However, what occurs is that the quantum of energy input first transfers to very short wavelength disturbances that have a high spatial energy intensity. The energy is then re-distributed to waves of lower spatial energy intensity 
with an accompanying amplitude growth that maintains constant total mechanical energy of the panel. This type of amplitude adjustment for neutrally-stable linear waves on flexible surfaces has been demonstrated and explained in [19]. In the later sequence of time-steps of Fig. 9b these disturbances rapidly evolve into the dominant low-frequency divergence-instability mode predicted by the eigenvalue analysis of Fig. 7a as the infinite-time response.

Crighton \& Oswell [11] studied a similar problem comprising an fluid-loaded, infinitely long, elastic plate subjected to continuous line excitation. Their theoretical analysis predicted the existence of an absolute instability at $U^{\prime}=0.074$, a much higher speed than that (0.0151) used to generate the instability that evolves through Fig. 9. However, Crighton \& Oswell remarked that in an unpublished report by D. Atkins (cited by [7] and [10]) the presence of some structural damping precipitated an absolute instability that persisted down to zero flow speed for a plate of infinite length. Lucey [14] and Abrahams \& Wickham [13] subsequently demonstrated, for a long but finite elastic plate and an infinitely long damped plate respectively, that the Atkins absolute instability existed at the maxima turning point on the lower branch of the wavenumber-frequency dispersion curve and was therefore different to Crighton \& Oswell's 'triple-point' absolute instability. The divergence instability in the present work aligns better with the Atkins instability of an infinitely long flexible plate but does not persist down to a zero critical flow speed because, as demonstrated by Fig. 9, it is the finite length of the panel that determines the longest permissible disturbance wavelength and thus the non-zero value of critical flow speed.

Figure 10 shows the transient response at flow speed $U^{\prime}=0.0227$ that is predicted to give the modal-coalescence flutter instability of Fig. 8. Figures 10a and 10b show the early response to the initial excitation as a sequence of wall-deformation plots within the time ranges $1 \Delta T^{\prime}$ to $50 \Delta T^{\prime}$ and $50 \Delta T^{\prime}$ to $2,500 \Delta T^{\prime}$ respectively where $\Delta T^{\prime}=0.813$. At later times in the evolution Fig. 10c shows superimposed panel profiles as time progresses for the time range 2,500 $\Delta T^{\prime}$ to $125,00 \Delta T^{\prime}$, in equal time steps of $2,500 \Delta T^{\prime}$, that follow immediately from that of Fig. 10b. Figure 10a shows that essentially flexural waves of the plate carry disturbance energy away from the source of excitation in both upstream and downstream directions with a similar phenomenology 
to that in the establishment of divergence instability described in the preceding paragraph. After reflection from the end points wave superposition occurs and longer wavelength disturbances start to dominate the response as seen in Fig. 10b. Finally, in Fig. 10c, the highly unstable flutter mode becomes established and rapid deformation growth is evidenced. For even later times of the same simulation, Fig. 10d shows a sequence of responses over one cycle of oscillation $\left(200,000 \Delta T^{\prime}\right.$ to $220,000 \Delta T^{\prime}$, in equal time steps of $\left.730 \Delta T^{\prime}\right)$ to highlight the mode shape of the instability; this is seen to be exactly the same as the infinite-time prediction of Fig. 8a.

Thus, we have mapped out the entire evolution history of divergence instability and modalcoalescence flutter when initiated by a line impulse. The mechanism for disturbance spread at early times means that a similar process of panel destabilisation would occur if the initial excitation were applied at a position on the panel other than its mid-point. Overall, the route to instability closely resembles that termed 'from waves to modes' in the studies of [25] for fluid-conveying flexible pipes. Given that high-frequency (lightly-loaded) flexural waves feature significantly in the early development of both divergence and flutter instabilities, the effects of dissipation in the structure and/or designing-in structural damping, for example through doping the panel, could serve to inhibit the development of instability at post-critical flow speeds.

We now show the extent to which the strategy of divergence postponement by an added spring support can be taken. Figures 11a and 11b show the variation of flow speeds of divergence onset, divergence recovery and model-coalescence flutter onset with the magnitude of the added spring support, $k_{s}^{\prime}$ for two cases of spring location $x_{k} / L=0.5$ and 0.25 . For ease of interpretation it is more convenient to non-dimensionalise distance of the added-spring location from the leading edge by the panel length. For each figure $L^{\prime}=92.31$, the same used throughout this sub-section and we note that the critical speeds plotted take the functional form

$$
U_{c}^{\prime}=f\left(L^{\prime}, k_{s}^{\prime}, x_{k} / L\right)
$$

These results clearly show that the addition of a single localised spring support can significantly increase the divergence-onset flow speed. As could be expected on physical grounds, this strategy 
is more effective when the spring is placed at the panel mid-point. When placing it here, it is noted that there is a threshold of approximately $k_{s}^{\prime}=0.4 \times 10^{-3}$ for which further stabilization of the system ceases. This is because the second system mode replaces the first as the critical mode for divergence onset, the spring support being so stiff that it effectively divides the original panel into two separate panels of equal length. Accordingly, this value may be regarded as optimal for the design of divergence-free flexible panels.

The results presented herein show that the addition of an isolated spring support to the structure can yield a very significant extension to the flow-speed range of a simple flexible panel before divergence instability sets in. To give an engineering feel for this benefit, we provide the following dimensional examples that arise from the non-dimensional results. For aluminium panels subjected to a water flow (i.e. defining the properties of the two media) with the single spring added at the panel mid-point, the functional relation of Eqn. (19) with divergence onset as the critical speed takes the dimensional form $U_{D}=f\left(L / h, k_{s}, 0.5\right)$. We consider three aluminium panels typically used for the hulls of high-speed ships. These have lengths and thicknesses: (i) $L=0.6 \mathrm{~m}, h=2.5$ mm giving $L / h=240$, (ii) $L=1.2 \mathrm{~m}, h=8.0 \mathrm{~mm}$ giving $L / h=150$, and (iii) $L=0.9144 \mathrm{~m}$, $h=6.35 \mathrm{~mm}$ giving $L / h=144$. Table 1 shows the predicted divergence-onset flow speed for each of these panels and how this increases with the addition and stiffening of an added spring support. Note that these predictions from our two-dimensional analysis are lower than those for a truly finite panel held along all four of its edges. For example, [4] shows that a panel with an aspect ratio 1 (width divided by length), has a divergence-onset flow speed that is approximately twice that predicted by the two-dimensional (infinite aspect ratio) analysis herein, whereas at aspect ratio 5 the difference is approximately $5 \%$. However, the relative increases to onset flow speeds achieved by adding a single spring support would also be expected for truly finite panels. The data of Table 1 shows how the addition of a spring support can increase the operational speed of a craft. Alternatively, the operational flow-speed limit imposed by hydroelastic instability can be kept constant and a spring support added to permit a thinner panel to be used. Table 2 therefore shows how the thicknesses of the three panels could be reduced if a spring support were to be introduced 
to keep the divergence-onset flow speed unchanged. While these results indicate material, hence mass, savings, the addition of a transverse array of spring imposes a mass penalty. To quantify this balance, we consider Panel (ii) in Table 2. The panel mass saved, per transverse metre, by reducing its thickness is 3.12 and $5.93 \mathrm{~kg} / \mathrm{m}$ respectively when introducing added spring support at $6 k_{+}$and $15 k_{+}$. Suitable transverse arrays of springs would respectively have masses $0.425 \mathrm{~kg} / \mathrm{m}(18$ springs per metre with maximum load capacity $1321 \mathrm{~N} / \mathrm{m}$ ) and $1.062 \mathrm{~kg} / \mathrm{m}$ (45 springs per metre with maximum load capacity $3301 \mathrm{~N} / \mathrm{m}$ ). Thus, the net mass savings, relative to an unsupported panel, when adding spring support at $6 k_{+}$and $15 k_{+}$, would then respectively be $11 \%$ and $19 \%$ which remain a significant engineering benefit.

\subsection{Flexible panel with a single-spring support: aeroelastic applications}

When considering the non-dimensional FSI system defined by Eqns. (12)-(14) it is evident that the flow speeds of divergence onset, divergence recovery and modal-coalescence flutter onset are, for a simple unsupported elastic panel, functions of the non-dimensional length, $L^{\prime}$ that is the inverse of the solid-to-fluid mass ratio. Sections 3.1 and 3.2 have primarily used $L^{\prime}=70.4$ and 92.3 respectively. We now present the FSI phenomenology of a panel with the much lower value of $L^{\prime}=0.225$ that we will show is markedly different from that prevailing at the higher value used in Section 3.2. This finding is of practical relevance because such results appertain to the aero-elasticity of a typical glass panel of a curtain wall subjected to an axial-flow wind loading with air density $\rho=1.27 \mathrm{~kg} / \mathrm{m}^{3}$. To illustrate how this non-dimensional data could be constituted, the glass panel would have $h=0.004 \mathrm{~m}, L=1.7 \mathrm{~m}, \rho_{m}=2400 \mathrm{~kg} / \mathrm{m}^{3}$, and $B=381 \mathrm{Nm}$ (having used $E=6.85 \times 10^{10} \mathrm{~N} / \mathrm{m}^{2}$ and $\left.\nu=0.2025\right)$. When we investigate the same stabilisation strategy used in Section 3.2, the added spring has stiffness $k_{s}=n k_{+}$given in multiples, $n$, of $k_{+}=8.5 \times 10^{3}$ $\mathrm{N} / \mathrm{m}^{2}$ that when non-dimensionalised gives $k_{+}^{\prime}=9.64 \times 10^{3}$.

Figure 12 shows the variation of the two lowest system eigenvalues with flow speed for the standard panel and two cases of a panel with an additional spring support, $k_{s}^{\prime}=1 k_{+}^{\prime}$ and $2 k_{+}^{\prime}$, located at its centre. We first focus upon the standard case, comparing the solution morphology 
with Fig. 4 for which the fluid was water. As the air speed is increased divergence-onset is first encountered at $U^{\prime}=59.35$; combining this with the present $L^{\prime}=0.225$ through Eqn. (15) gives the non-dimensional stiffness ratio $\Lambda=40.1$ for divergence-onset, again in excellent agreement with previous works as discussed in Section 3.2. Beyond this critical speed the panel undergoes Mode-1 divergence. Further increases to $U^{\prime}$ then cause Mode 2 to succumb to divergence instability at $U^{\prime}=155.0$. In a physical situation Mode-1 divergence would be seen to dominate the panel behaviour because it has a higher amplification rate. The hydroelastic result of Fig. 4 shows Mode-1 divergence recovery with neutral stability of Mode 2 until a further increase to flow speed causes these two oscillatory modes to coalesce into flutter. In contrast, the airflow case features the coalescence of the two unstable non-oscillatory divergence Modes 1 and 2 to create the oscillatory flutter instability. The fact that two non-oscillatory modes can merge to give an oscillatory instability is highly unusual, although we have shown earlier in Fig. 7a that, although a divergence eigenmode has zero oscillatory component, its form of amplification gives it the character of a travelling wave; this was first proposed in [16]. The explanation for the unusual overall behaviour is that for airflow the FSI is dominated by fluid-stiffness effects due to the third term ( $\rho U_{\infty}^{2}$-dependent) of the fluid-pressure loading in Eqn. (6). To generate equivalent hydrodynamic-stiffness effects between air (with density $\rho_{A}$ ) and water (with density $\rho_{W}$ ) flows, the fluid-damping and fluid inertia effects, respectively given by the second and first terms in the right-hand side of Eqn. (6), are factors of $\sqrt{\rho_{A} / \rho_{W}}$ and $\rho_{A} / \rho_{W}$ smaller for air than water. When water is the fluid medium the magnitude of these terms is principally responsible for the coupling of Modes 1 and 2 that leads to the far-better known sequence of divergence recovery followed by modal-coalescence flutter.

The foregoing discussion shows that the fluid density and, in particular, its value relative to that of the solid medium is responsible for the solution morphology remarked upon in the standard case of Fig. 12. In the non-dimensional scheme, the panel length $L^{\prime}$, defined in Eqn. (14a), may be interpreted as the fluid-to-solid density ratio for a panel of given dimensional length and thickness. We therefore show how the key flow-speeds of Mode-1 divergence onset, Mode-1 divergence recovery or Mode-2 divergence onset, and modal-coalescence flutter vary with $L^{\prime}$ in Fig. 13. Rather than 
flow speed, we plot $\left(U^{\prime}\right)^{2}\left(L^{\prime}\right)^{3}$ which is the fluid-to-solid stiffness, $\Lambda$, on the vertical axis against the logarithm of $1 / L^{\prime}$ on the horizontal axis because the domain covers number of decades. First we remark that Fig. 12 indicates that the value of $\Lambda$ for Mode- 1 divergence onset is independent of $L^{\prime}$ taking the value 40.1. This is to be expected because exactly at divergence onset the panel is static and the solid inertia (and thus density) can play no part in its determination. For post-Mode-1 divergence behaviour, Fig. 13 shows that the system solution follows the pattern typical of water over a metal panel for $1 / L^{\prime}$, inversely proportional to the fluid density, up to a threshold value of approximately 0.57 (noting that $\ln (0.57)=-0.57$ on the horizontal scale). For higher values than this, the fluid density is sufficiently low, relative to that of the solid, that both Modes 1 and 2 concurrently succumb to divergence instability and then coalesce at higher flow speeds to create the flutter instability. This interpretation is based upon density differences between fluid and solid media. The alternative interpretation of Fig. 13 is that, for given solid and fluid media, a panel with low $h / L$ (long panel for a given thickness) has the well-known solution morphology following the sequence of Mode-1 divergence-onset, Mode-1 divergence recovery, modal-coalescence flutter typified by Fig. 4, whereas a panel with high $h / L$ (short panel for a given thickness) evinces the unusual two-mode divergence and then coalescence route to flutter typified by Fig. 12 .

We now return to Fig. 12 to consider the effect of adding a spring support to the panel as a stabilisation strategy. It is seen that the added spring postpones Mode- 1 divergence onset to higher flow speeds. It does not affect Mode-2 divergence onset because the spring has been added at the panel mid-point where there is a quasi-node for this mode. With regard to post-divergence modal-coalescence flutter, Fig 12 shows that increasing the spring-stiffness coefficient reduces the flutter-onset speed slightly and causes the envelope of the amplifying part of its eigenvalue to narrow with increasing flow speed. In Fig. 14 we show the effect of further increases to the spring-stiffness coefficient on the solution morphology. Figures $14 \mathrm{a}$ and $14 \mathrm{~d}$ are obtained when $k_{s}^{\prime}=1.5 k_{+}^{\prime}$ and show results similar to those shown in Fig. 12. When $k_{s}^{\prime}=2.5 k_{+}^{\prime}$, Figs. $14 \mathrm{~b}$ and $14 \mathrm{e}$ show a clear difference. At flow speeds higher than the range that gives modal-coalescence flutter, the system decouples and returns to Mode- 1 and Mode- 2 divergence at $U^{\prime} \approx 240$. Figures $14 \mathrm{c}$ and $14 \mathrm{f}$ show 
that when $k_{s}^{\prime}=3.5 k_{+}^{\prime}$ the modal-coalescence flutter disappears entirely and the system stability for the range of flow speeds shown is dominated by Mode-2 divergence. The continuous variation of divergence- and flutter-onset flow speeds with the spring-stiffness coefficient, $k_{s}^{\prime}$ is shown in Figs. $15 \mathrm{a}$ and $15 \mathrm{~b}$ with the spring located at $x_{k} / L=0.5$ and 0.25 respectively. The critical speeds are the loci of the function written as Eqn. (19) with $L^{\prime}=0.225$ for the present data. With the spring located at the panel mid-point it is seen that the change of solution morphology occurs at $k_{s}^{\prime}=2.69 \times 10^{4}\left(=2.75 k_{+}\right)$with the replacement of modal-coalescence flutter by two-mode divergence illustrated by the sequence in Fig. 14. Thus, the inclusion of an isolated spring can be used the replace a more damaging dynamic instability such as flutter with divergence buckling of the panel that would become static due to nonlinear saturation if even a marginal level of structural damping were present $([17,26])$. It is also noted that both Figs. $15 \mathrm{a}$ and $15 \mathrm{~b}$ indicate that the onset of divergence can be postponed to significantly higher flow speeds through the addition of an isolated spring. Clearly the postponement is greatest when the spring is located at the panel mid-point because it is Mode 1 that gives the onset of divergence below the threshold value of $k_{s}^{\prime}=2.69 \times 10^{4}$ in Fig. 15a and throughout the range of $k_{s}^{\prime}$ for Fig. 15b.

To give an engineering feel for the potential benefits of stabilisation through an added spring support, Table 3 provides dimensional examples of critical wind speeds for glass panels of different lengths and thicknesses. The glass panel has a single spring added at its mid-point and, with the two media defined, the critical-speed function of Eqn. (19) for divergence-onset becomes $U_{D}=$ $f\left(L / h, k_{s}, 0.5\right)$. Table 3 lists the predicted divergence-onset flow speeds (in $\mathrm{km} / \mathrm{h}$ ) for each of three typical flat tempered-glass panels for different values of the stiffness coefficient of the added spring. Further increases to spring-stiffness would yield even higher divergence-onset wind speeds because, for example, the results for the panel with $L / h=406$ that has a mass ratio similar to that used to generate Fig. 15a approximately correspond to data points on the rising divergence-onset curve between $k_{s}^{\prime}=0$ and $0.5 \times 10^{4}$. Given that Category 1 cyclones have wind speeds in the range 119-153 km/h, while Category 4 and 5 cyclones respectively generate wind-speeds in the ranges 210-249 km/h and over $250 \mathrm{~km}$, the value of this stabilisation strategy for the panels of curtain 
walls is clearly evident.

\section{CONCLUSIONS}

We have extended the methods of [15] to study the initial-value problem of a finite flexible panel or compliant wall interacting with an inviscid flow for small (linear) disturbance amplitudes. Our results show that waves propagate in both downstream and upstream directions from a highly localised source of excitation so that with the passage of time instability comes to occupy all locations of the flexible panel. Thus, at applied flow speeds greater than those of divergence and modal-coalescence flutter, destabilisation is global and ultimately leads to the establishment of the system eigenmodes that are predicted by the boundary-value problem. This elucidation of the transients that lead to system instability may permit the design of intervention strategies to postpone, either in developmental time or to a higher critical flow speed, the onset of instability.

By investigating the solution space over a range of non-dimensional mass ratios, we have shown that different eigen-system morphologies can exist. Of particular note is that, when the fluid density is much lower than that of the solid for given panel dimensions (e.g. a wind flow over a realistic glass panel), flutter instability occurs through the coalescence of two non-oscillatory divergence modes. This counter-intuitive behaviour can be understood by recognising that at post-divergence flow speeds, divergence modes evidence a form of downstream wave travel more usually identified with conventional oscillatory travelling waves. A similar phenomenology can occur for denser fluids when the panel is very short for which high flows speeds are required for its destabilisation. The instability phase space has been mapped out to identify quantitatively the value of mass ratio at which the flutter-onset mechanism switches between divergence-mode coalescence and oscillatorymode coalescence.

The major finding of the present work is that the addition of highly localised stiffening to the structural design of an otherwise homogeneous flexible panel can be a very effective means to postpone instability to a higher flow speed or beneficially modify the form of instability. Divergence

postponement can be achieved across the full range of mass ratios, most effectively through the 
addition of an isolated spring support at the panel mid-chord. A stiffer spring yields a greater postponement until an optimal value of spring stiffness is reached at which the critical mode switches from Mode 1 to Mode 2 and no further postponement of divergence occurs. This type of tailored stabilization strategy may find engineering use in that it can be far more effective than a 'brute force' approach to design that, for example, thickens the entire panel to prevent aero-/hydro-elastic instability within the envelope of operational flow speeds. For applications with low values of mass ratio, the addition of an isolated spring has also been shown to change the eigen-system morphology so that modal-coalescence flutter instability is replaced by two-mode divergence. This can be advantageous because flutter is a dynamic instability leading to material fatigue whereas divergence instability grows into a static nonlinear buckled state.

Stabilisation by means of an added spring is more effective than including an additional fixed (zero-displacement) restraint within the panel streamwise extent as investigated in [15]. A fixed restraint may be considered an added spring of infinite stiffness in the context of the present work and this exceeds the optimal value of spring-stiffness for divergence postponement. Moreover, [15] showed that a fixed restraint modifies the spatial energy balance of the panel and can introduce a new low-speed form of mild panel flutter. This does not occur in the present work that permits motion of the spring's attachment point to the panel.

Finally, for real engineering applications, the use of an added spring in the present two-dimensional work is questionable because the lower end of the spring must be attached to a rigid structure that could equally be used as a support to replace the panel with two shorter, more stable, flexible panels. However, in the three-dimensional application of localised stiffening, the spring would be replaced by a transverse stiffening strip adhered to the under-surface of the panel and attached to the side edges of the ribbed bay or baffle that the panel encloses. Our preliminary work on this extension, [27], shows that the stabilising benefits demonstrated in the present paper carry across into the full three-dimensional problem thereby making the localised stiffening strategy a practicable technology. Moreover, in the full problem multiple combinations of both transverse and streamwise stiffening strips could be used to optimise instability postponement for a given overall 
- plate plus stiffening strips - structural mass.

\section{ACKNOWLEDGEMENTS}

The authors gratefully acknowledge the many valuable discussions with Dr M.W. Pitman that have contributed to the work of this paper and the Australian Research Council through the grant DP1096376 supporting the project of which the present work forms a part. 


\section{References}

[1] Dugundji, J., Dowell, E. \& Perkin, B., Subsonic flutter of panels on a continuous elastic foundation. AIAA Journal 1, pp. 1146-1154, 1963.

[2] Weaver, D.S. \& Unny, T.S., The hydroelastic stability of a flat plate. ASME: Journal of Applied Mechanics 37, pp. 823-827, 1971.

[3] Ellen, C.H., The stability of simply supported rectangular surfaces in uniform subsonic flow. ASME: Journal of Applied Mechanics 95, pp. 68-72, 1973.

[4] Lucey, A.D. \& Carpenter, P.W., The hydroelastic stability of three-dimensional disturbances of a finite compliant panel. Journal of Sound and Vibration 165, pp.527-552, 1993.

[5] Guo, C. Q. \& Païdoussis, M. P., Stability of rectangular plates with free side-edges in twodimensional inviscid channel flow. Journal of Applied Mechanics 67, pp. 171-176, 2000.

[6] Benjamin, T.B., The threefold classification of unstable disturbances in flexible surfaces bounding inviscid flows. Journal of Fluid Mechanics 16, pp. 436-450, 1963.

[7] Carpenter, P.W. \& Garrad A.D., The hydrodynamic stability of flows over Kramer-type compliant surfaces. Part 2. Flow-induced surface instabilities. Journal of Fluid Mechanics 170, pp. 199-232, 1986.

[8] Lucey, A.D. \& Carpenter, P.W., On the difference between the hydroelastic instability of infinite and very long compliant panels. Journal of Sound and Vibration 163(1), pp. 176$181,1993$.

[9] Peake, N., On the unsteady motion of a long fluid-loaded elastic plate with mean flow. Journal of Fluid Mechanics 507, pp. 335-366, 2004.

[10] Brazier-Smith, P.R. \& Scott, J.F., Stability of fluid flow in the presence of a compliant surface. Wave Motion 6, pp. 436-450, 1984.

[11] Crighton, D.G. \& Oswell, J.E., Fluid loading with mean flow. I. Response of an elastic plate 
to localized excitation. Philosophical Transactions of the Royal Society of London A 335, pp. 557-592, 1991.

[12] Peake, N., On the behaviour of a fluid-loaded cylindrical shell with mean flow. Journal of Fluid Mechanics 338, pp. 387-410, 1997.

[13] Abrahams, I.D. \& Wickham, G.R., On transient oscillations of plates in moving fluids. Wave Motion 33, pp. 7-23, 2001.

[14] Lucey, A.D., The excitation of waves on a flexible panel in a uniform flow. Philosophical Transactions of the Royal Society of London A 356, pp. 2999-3039, 1998.

[15] Pitman, M.W. \& Lucey, A.D., On the direct determination of the eigenmodes of finite flowstructure systems. Proceedings of the Royal Society A 465, pp. 257-281, 2009.

[16] Lucey, A.D. \& Carpenter, P.W., A numerical simulation of the interaction of a compliant wall and inviscid flow. Journal of Fluid Mechanics 234, pp. 121-146, 1992.

[17] Lucey, A.D., Cafolla, G.J., Carpenter, P.W. \& Yang, M., The nonlinear hydroelastic behaviour of flexible walls. Journal of Fluids and Structures 11, pp. 717-744, 1997.

[18] Garrad, A.D. \& Carpenter, P.W., A theoretical investigation of flow-induced instabilities in compliant coatings. Journal of Sound and Vibration 84(4), pp. 483-500, 1982.

[19] Lucey, A.D., Sen, P.K. \& Carpenter, P.W., Excitation and evolution of waves on an inhomogeneous flexible wall in a mean flow. Journal of Fluids and Structures 18, pp. 251-267, 2003.

[20] Carpenter, P.W. \& Garrad A.D., The hydrodynamic stability of flows over Kramer-type compliant surfaces. Part 1. Flow instabilities. Journal of Fluid Mechanics 155, pp. 465-510, 1985.

[21] Landahl, M.T., On the stability of a laminar incompressible boundary-layer over a flexible surface. Journal of Fluid Mechanics 13, pp. 609-632, 1962. 
[22] Huerre, P. \& Monkewitz, P.A., Local and global instabilities in spatially developing flows. Annual Review of Fluid Mechanics 22(1), pp. 473-537, 1990.

[23] Lucey, A.D. \& Peake, N., Wave excitation on flexible walls in the presence of a fluid flow. In IUTAM: Flow through collapsible tubes and past other highly compliant boundaries (eds. P.W. Carpenter \& T.J. Pedley), Kluwer Academic Publishers, pp. 118-145, 2003.

[24] Ishii, T., Aeroelastic instabilities of simply supported panels in subsonic flow. Meeting of the American Institute of Aeronautics and Astronautics, Los Angeles Paper AIAA-65-752, 1965.

[25] Doaré, O. \& De Langre, E., Local and global instability of fluid-conveying pipes on elastic foundations. Journal of Fluids and Structures 16(1), pp. 1-14, 2002.

[26] Ellen, C.H., The non-linear stability of panels in incompressible flow. Journal of Sound and Vibration 54(1), pp. 117-121, 1977.

[27] Tan, B.H., Lucey, A.D. \& Pitman, M.W., Stability of a structurally inhomogeneous flexible plate in uniform axial flow. In Proc. of the 10th International Conference on Flow Induced Vibration \& Flow-Induced Noise (eds. C. Meskell \& G. Bennett), pp. 203-210, 2012. 


\section{Table Captions}

TABLE 1: Examples of the increase to divergence-onset flow speed for typical aluminum panels forming the hull of a high-speed ship through the addition of a single spring support at the panel mid point. The length and thickness of the panels are respectively: (i) $L=0.6 \mathrm{~m}, h=2.5 \mathrm{~mm}$ giving $L / h=240$, (ii) $L=1.2 \mathrm{~m}, h=8.0 \mathrm{~mm}$ giving $L / h=150$, and (iii) $L=0.9144 \mathrm{~m}, h=6.35$ mm giving $L / h=144$.

TABLE 2: Examples of material savings (by decreasing panel thickness) keeping the divergenceonset flow speed unchanged for typical aluminium panels forming the hull of a high-speed ship through the addition of a spring support at the panel mid point. Panels (i)-(iii) as described in Table 1.

TABLE 3: Examples of the increase to divergence-onset flow speed for typical flat tempered-glass panels subjected to axial wind-flow through the addition of a single spring-support at the panel mid-point.

\section{Figure Captions}

FIGURE 1: Schematics of a uniform flow past a flexible panel with (a) uniformly distributed spring foundation, and (b) localised added spring support.

FIGURE 2: Variation of system eigenvalues with non-dimensional flow speed for a (plate-spring) compliant-wall panel with $L^{\prime}=422.5, d^{\prime}=11.30$ and $K^{\prime}=4.41$ : (a) is the real (positive, growth; negative, decay) part, and (b) is the imaginary (oscillatory) part of the eigenvalues.

FIGURE 3: Sequence of instantaneous panel profiles developing (top to bottom) from a line impulse applied at the mid-point for the compliant-wall panel of Fig. 1 at: (a) a marginally predivergence flow speed, $U^{\prime}=2.320$, at time steps $1 \Delta T^{\prime}, 5 \Delta T^{\prime}, 20 \Delta T^{\prime}, 50 \Delta T^{\prime}$, and $300 \Delta T^{\prime}$, and (b) a post-instability-onset flow speed, $U^{\prime}=6.410$, at time steps $125 \Delta T^{\prime}, 625 \Delta T^{\prime}, 1,250 \Delta T^{\prime}, 1,875 \Delta T^{\prime}$, and $2,500 \Delta T^{\prime}$, where $\Delta T^{\prime}=2.53$. 
FIGURE 4: Variation of the two lowest system eigenvalues, (a) real (positive, growth; negative; decay) part, and (b) imaginary (oscillatory) part, with non-dimensional flow speed for an elastic flexible panel with $L^{\prime}=92.31$ for different values of a spring support at the panel mid-point: — $0 k_{+}^{\prime}$ (homogeneous case); ——, $6 k_{+}^{\prime},-\triangle-, 15 k_{+}^{\prime}$, where $k_{+}^{\prime}=2.15 \times 10^{-5}$.

FIGURE 5: Variation of flexible-panel strain energy, $E_{S}(\cdots)$, kinetic energy, $E_{K}(+)$, spring energy, $E_{S P}(-)$ and virtual work done by the hydrodynamic stiffness, $E_{V W}$ (०) with time for neutrally stable oscillatory motion at a flow speed incrementally below that of divergence onset for the system of Fig. 4: (a) $0 k_{+}^{\prime}$ (homogeneous case), and (b) added spring support with coefficient $6 k_{+}^{\prime}$ added at the panel mid-point.

FIGURE 6: Snapshots of panel deformation illustrating the neutrally-stable oscillatory motion corresponding to the flexible-panel eigenmodes of Fig. 4 for the case of a spring support with $6 k_{+}^{\prime}$ at $U^{\prime}=0.00378$ : (a) Mode 1, (b) Mode 2, and (c) Mode 3. The thick broken line and circle symbols respectively indicate the starting and finishing deformations over the time period of the evaluation.

FIGURE 7: Snapshots of panel deformation illustrating motion in the divergence range of flow speeds of the flexible-panel eigenmodes of Fig. 4 for the case of a spring support with $6 k_{+}^{\prime}$ at $U^{\prime}=0.0151$ : (a) amplifying, and (b) decaying solutions of the divergence mode. The thick broken line and circle symbols respectively indicate the starting and finishing deformations over the time period of the evaluation.

FIGURE 8: Snapshots of panel deformation illustrating motion in modal-coalescence-flutter of the flexible-panel eigenmodes of Fig. 4 for the case of a spring support with $6 k_{+}^{\prime}$ at $U^{\prime}=0.0227$ : (a) showing modal amplification, and (b) with the modal growth suppressed to illustrate the mode shape. The thick broken line and circle symbols respectively indicate the starting and finishing deformations over the time period of the evaluation. 
FIGURE 9: Development of divergence instability from a line impulse applied at the mid-point of a flexible panel with spring support for the case of Figs. 4 (at $U^{\prime}=0.0151$ and with $6 k_{+}^{\prime}$ ) and 7: (a) sequence of instantaneous panel profiles (from top to bottom) at time steps $1 \Delta T^{\prime}, 10 \Delta T^{\prime}$, $20 \Delta T^{\prime}, 30 \Delta T^{\prime}$, and $50 \Delta T^{\prime}$, and (b) sequence of superimposed panel profiles for times $0 \Delta T^{\prime}$ to $250,000 \Delta T^{\prime}$ plotted at time increments of $2,500 \Delta T^{\prime}$, where $\Delta T^{\prime}=0.813$. In (b) the thick broken line and circle symbols respectively indicate the starting and finishing deformations over the time period of the evaluation.

FIGURE 10: Development of modal-coalescence flutter from a line impulse applied at the midpoint of a flexible panel with spring support for the case of Figs. 4 (at $U^{\prime}=0.0227$ and with $6 k_{+}^{\prime}$ ) and 8: (a) and (b) respectively show sequences $1 \Delta T^{\prime}, 6 \Delta T^{\prime}, 7 \Delta T^{\prime}, 20 \Delta T^{\prime}, 50 \Delta T^{\prime}$ and $50 \Delta T^{\prime}, 300 \Delta T^{\prime}, 350 \Delta T^{\prime}, 1000 \Delta T^{\prime}, 2,500 \Delta T^{\prime}$ of instantaneous panel profiles (from top to bottom in each and noting the different scales on the vertical axes), while thereafter (c) shows sequences of superimposed panel profiles for times $2,500 \Delta T^{\prime}$ to $125,000 \Delta T^{\prime}$ plotted at time increments of $2,500 \Delta T^{\prime}$, and (d) shows motion in the established mode for later times 200,000 $\Delta T^{\prime}$ to $220,000 \Delta T^{\prime}$ plotted at time increments of $730 \Delta T^{\prime}$, where $\Delta T^{\prime}=0.813$. In (c) and (d) the thick broken line and circle symbols respectively indicate the starting and finishing deformations over the time period of the evaluation.

FIGURE 11: Variation of divergence-onset (-o-), divergence recovery (- - -) and modalcoalescence flutter $(---)$ onset flow speeds with the coefficient of an added spring support for a flexible panel with $L^{\prime}=92.31$ : spring added at (a) panel mid-point $\left(x_{k} / L=0.5\right.$ ), and (b) $x_{k} / L=0.25$.

FIGURE 12: Variation of the two lowest system eigenvalues, (a) real (positive, growth; negative, decay) part, and (b) imaginary (oscillatory) part, with non-dimensional flow speed for an elastic flexible panel with $L^{\prime}=0.225$ for different values of a spring support at the panel mid-point: — $0 k_{+}^{\prime}$ (homogenous case); ——- $1 k_{+}^{\prime},-\triangle-, 2 k_{+}^{\prime}$, where $k_{+}^{\prime}=9.64 \times 10^{3}$.

FIGURE 13: Variation of Mode-1 divergence-onset (- --$)$, Mode-1 divergence recovery (- . $-)$, Mode-2 divergence-onset $(-\triangle-)$ ) and modal-coalescence flutter $(---)$ onset flow speeds $\left(\Lambda=\left(U^{\prime}\right)^{2}\left(L^{\prime}\right)^{3}\right)$ with the logarithm of the inverse of the mass ratio $L^{\prime}$ for a simple unsupported elastic panel. 
FIGURE 14: Variation of the two lowest system eigenvalues, (a), (b) and (c) real (positive, growth; negative, decay) part, and (d), (e) and (f) imaginary (oscillatory) part, with non-dimensional flow speed for an elastic flexible panel with $L^{\prime}=0.225$ for different values of a spring support at the panel mid-point: (a) and (d) $1.5 k_{+}^{\prime}$, (b) and (e) $2.5 k_{+}^{\prime}$, and (c) and (f) $3.5 k_{+}^{\prime}$, where $k_{+}^{\prime}=9.64 \times 10^{3}$.

FIGURE 15: Variation of Mode-1 divergence-onset (-o-), Mode-2 divergence-onset $(-\triangle-)$ and modal-coalescence flutter (- - ) onset flow speeds with the coefficient of an added spring support for a flexible panel with $L^{\prime}=0.225$ : spring added at (a) panel mid-point $\left(x_{k} / L=0.5\right)$, and (b) $x_{k} / L=0.25$. 


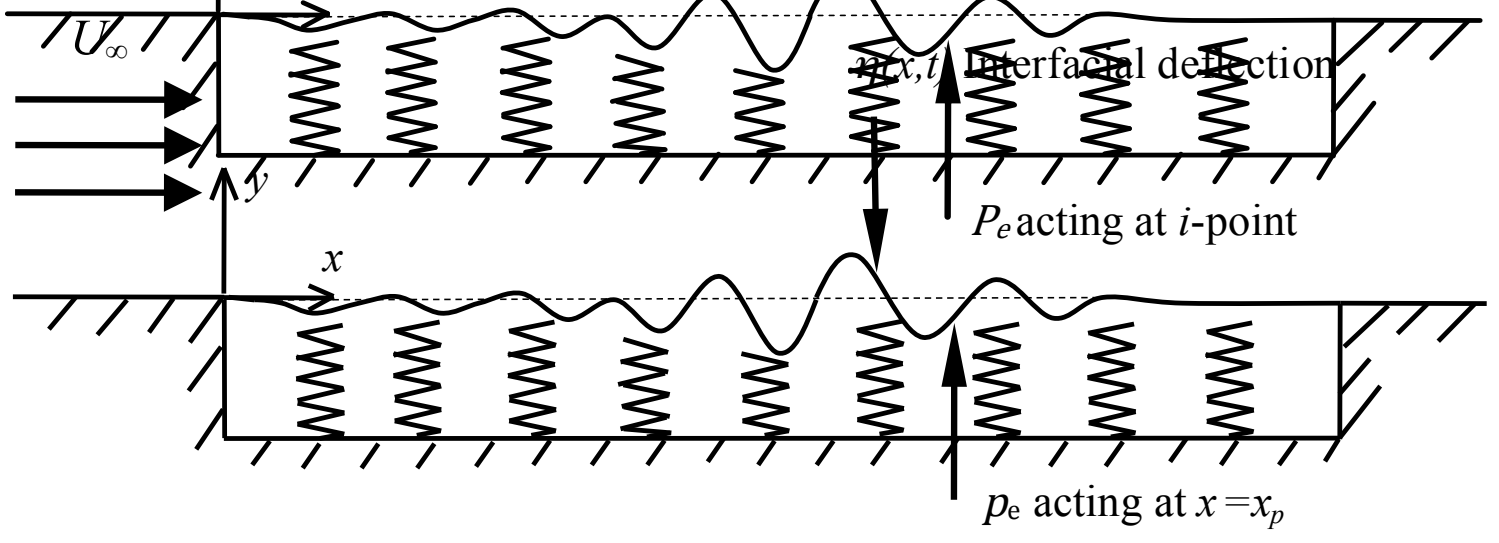

(a)

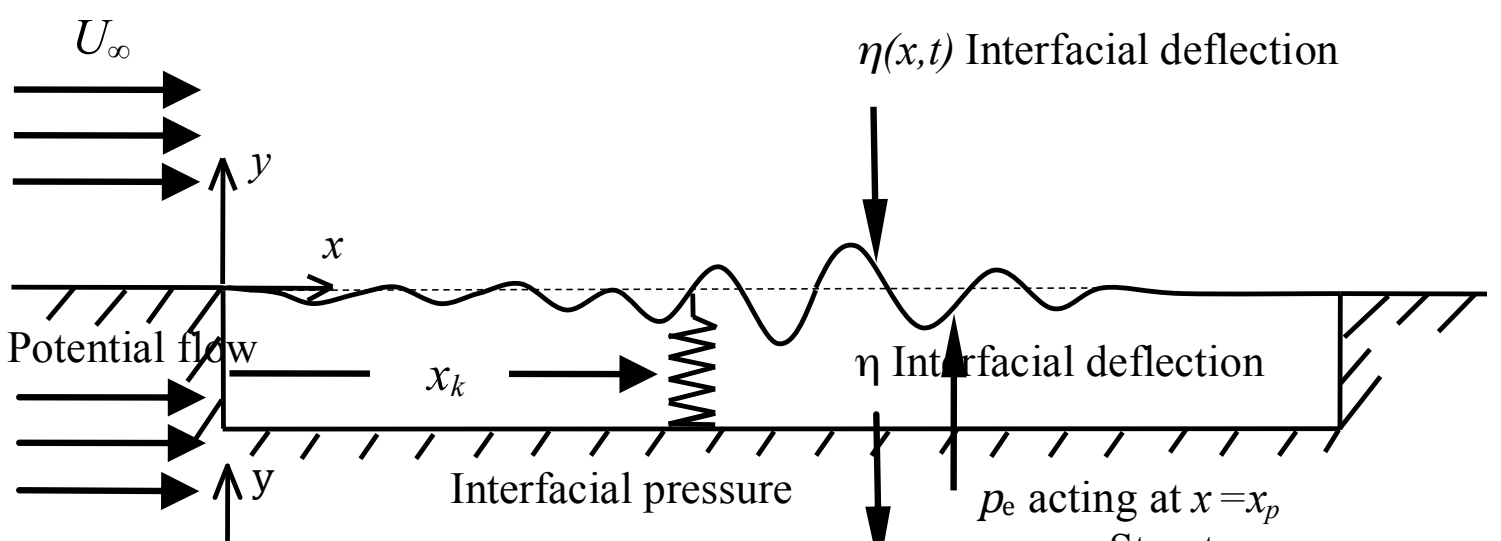

(b)

Figure 1: 


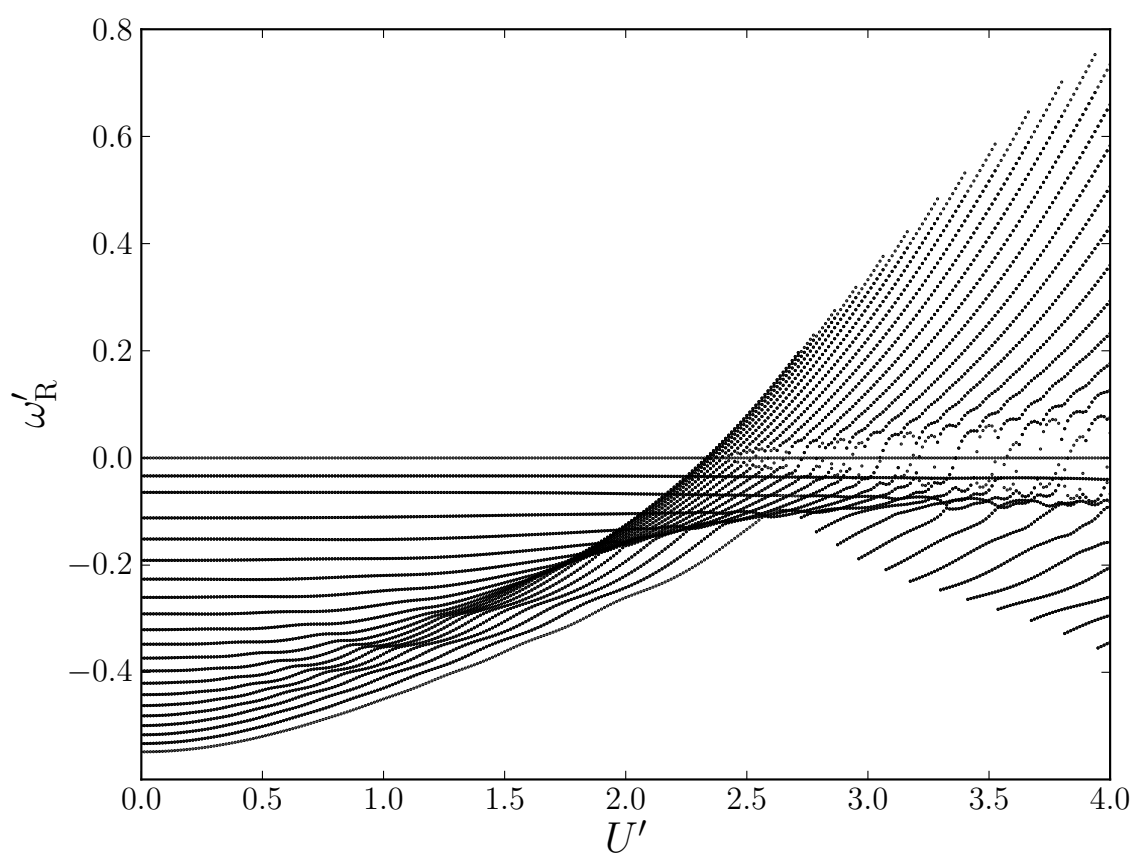

(a)

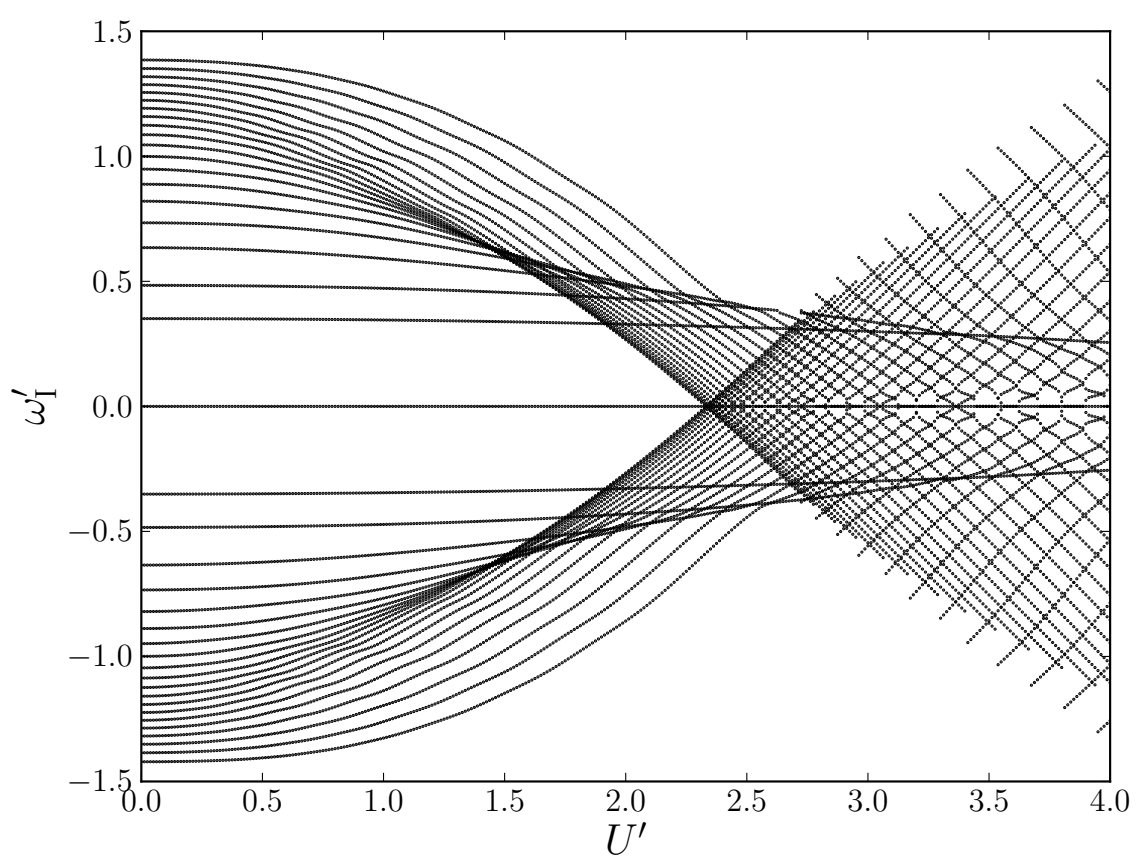

(b)

Figure 2: 


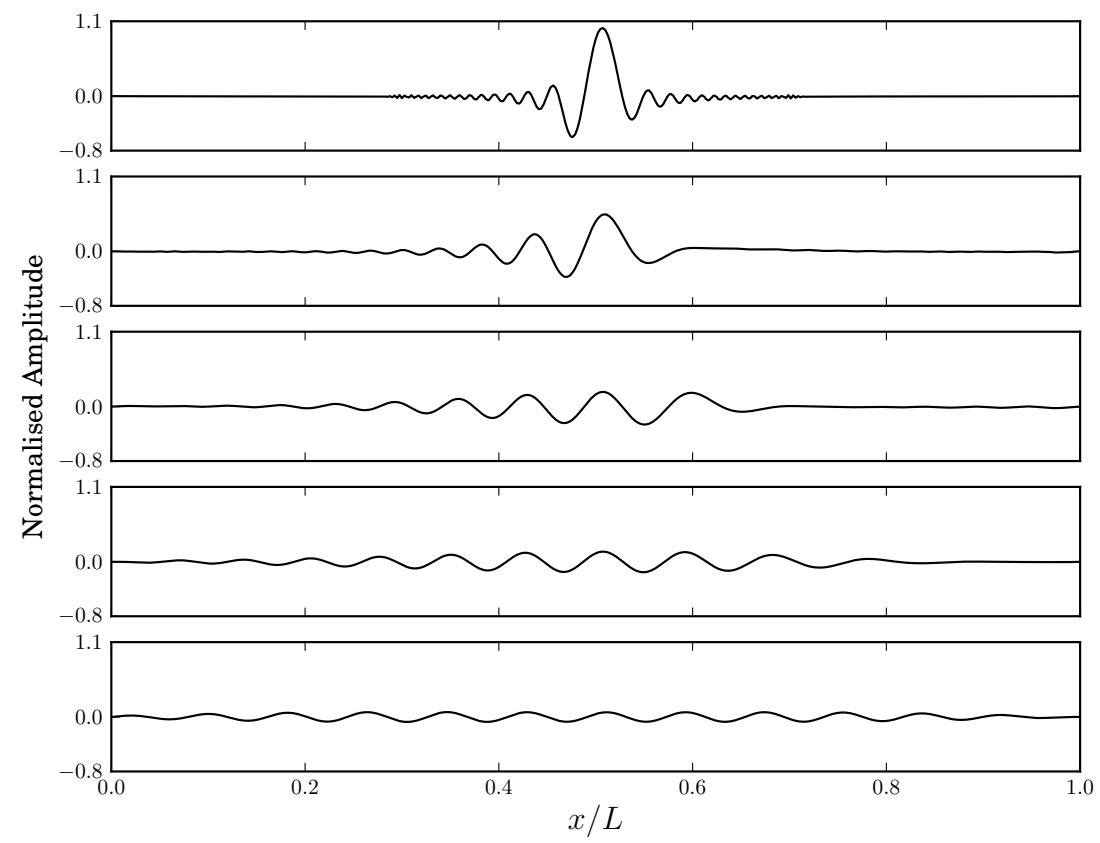

(a)

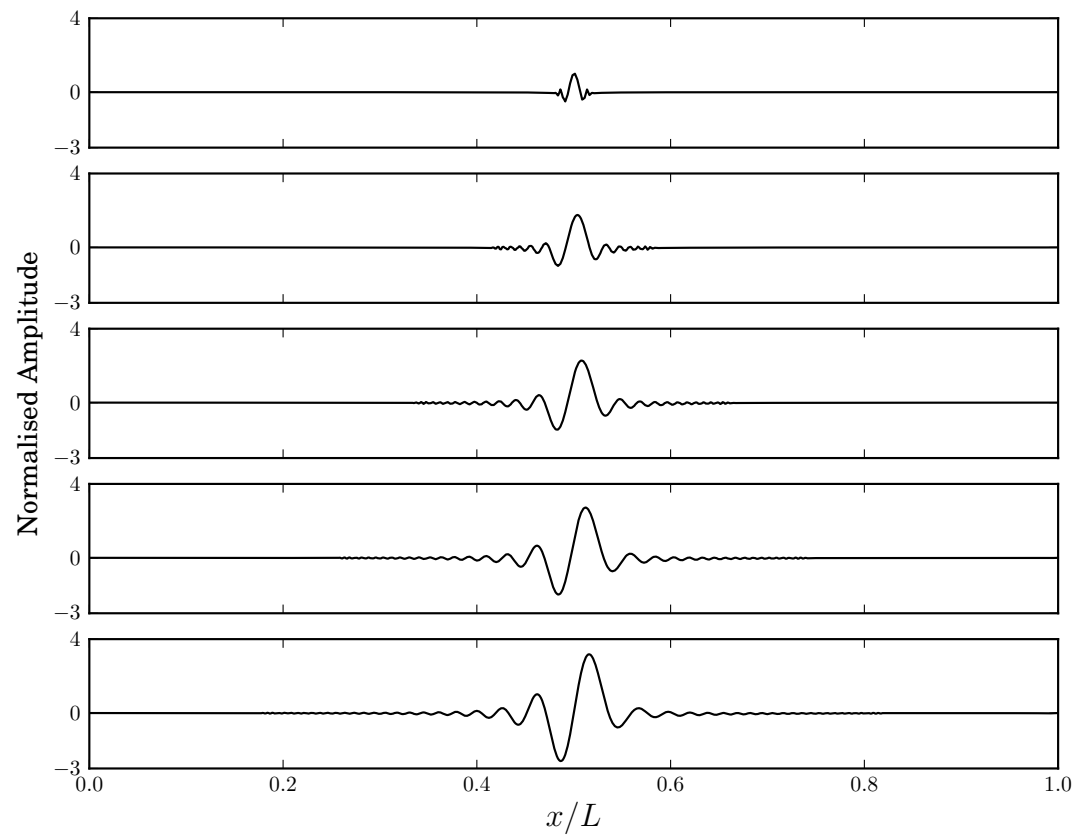

(b)

Figure 3: 


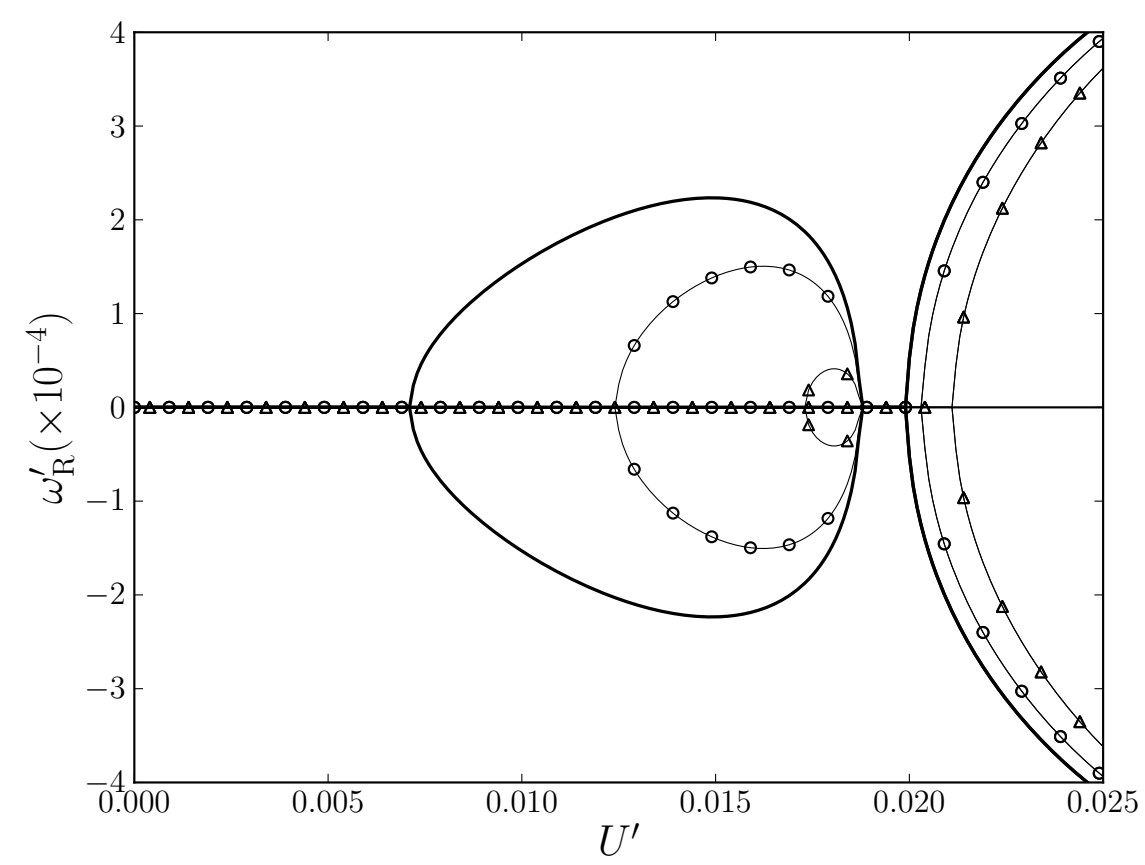

(a)

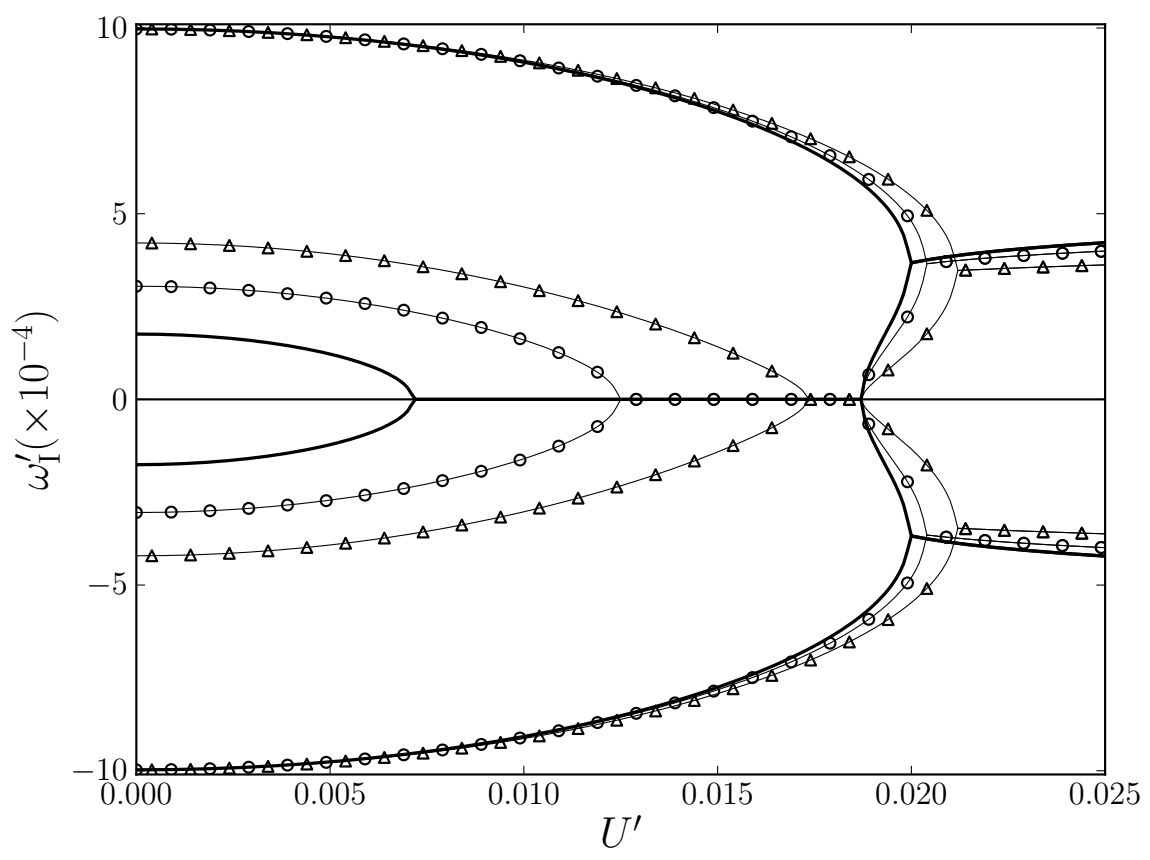

(b)

Figure 4: 


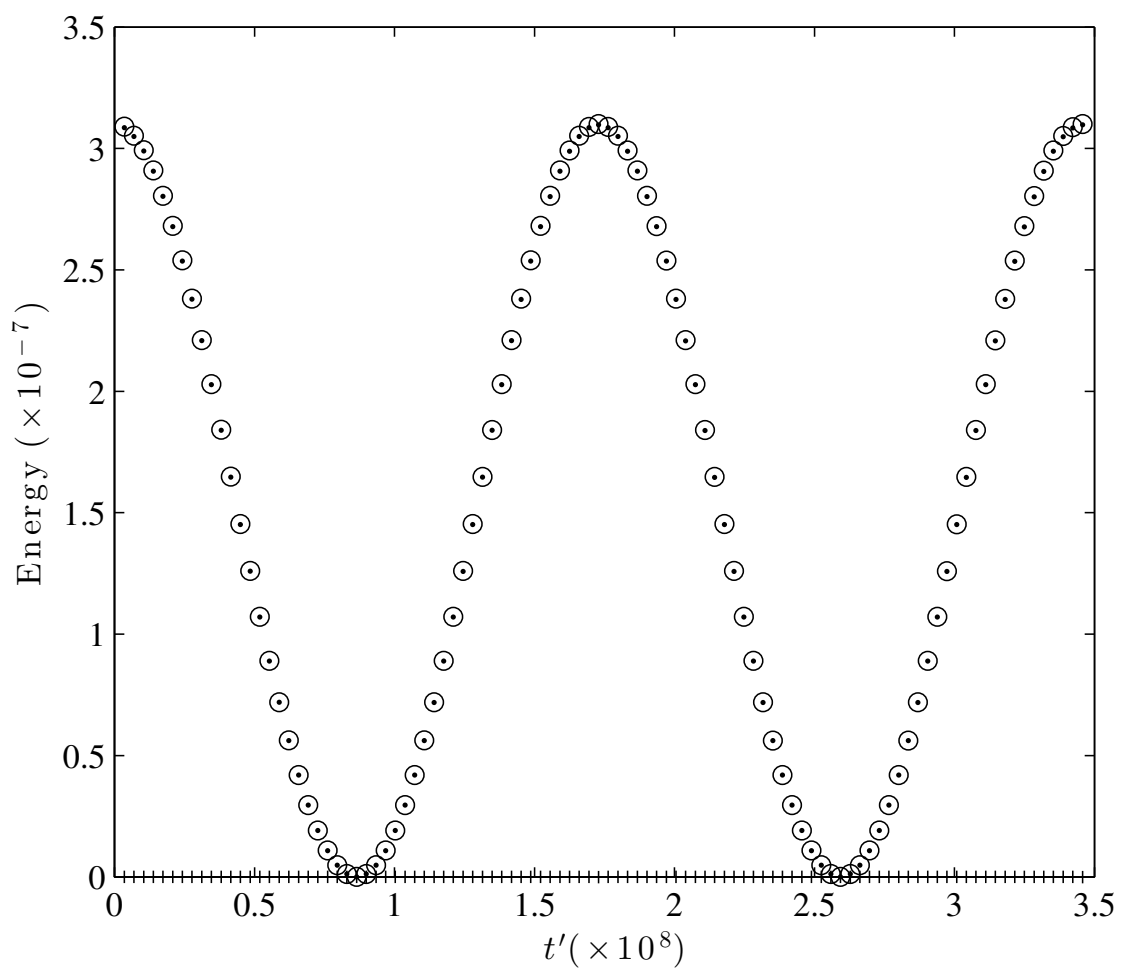

(a)

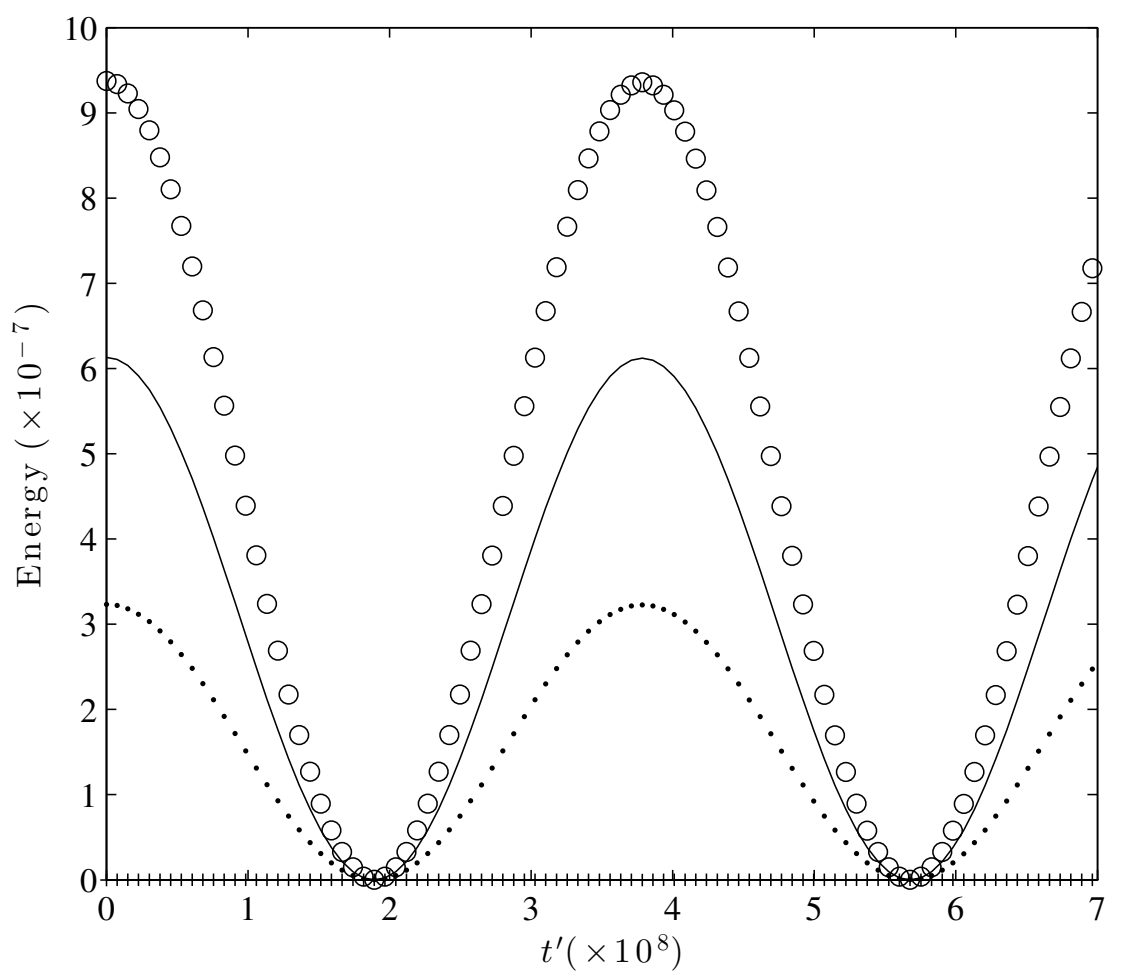

(b)

Figure 5: 


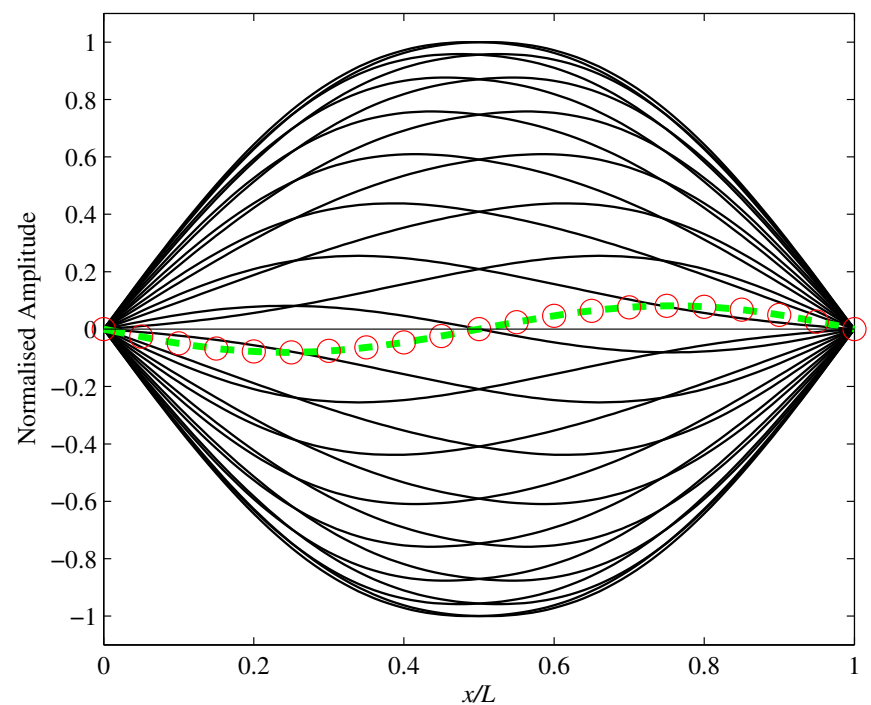

(a)

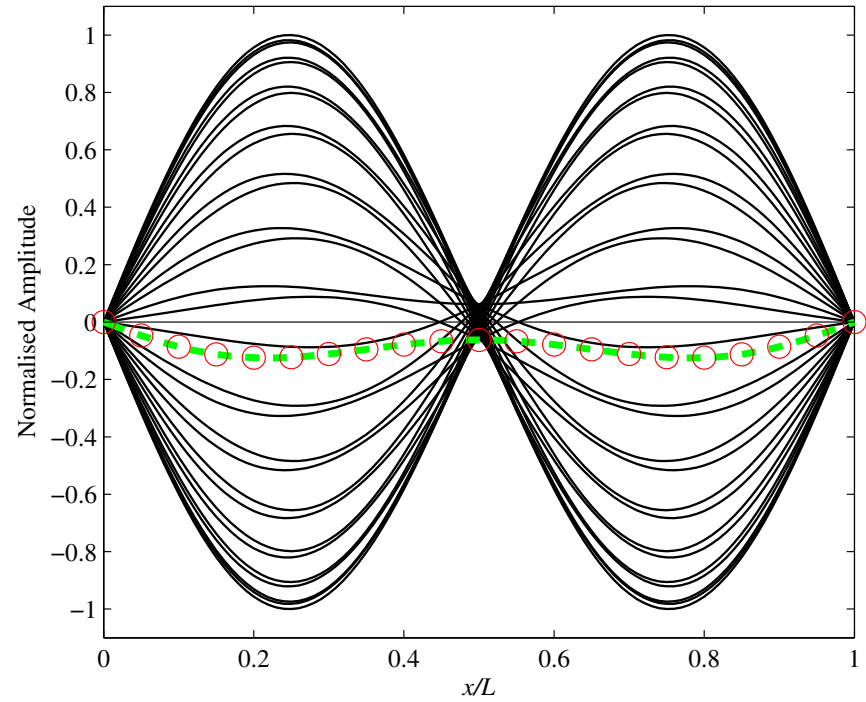

(b)

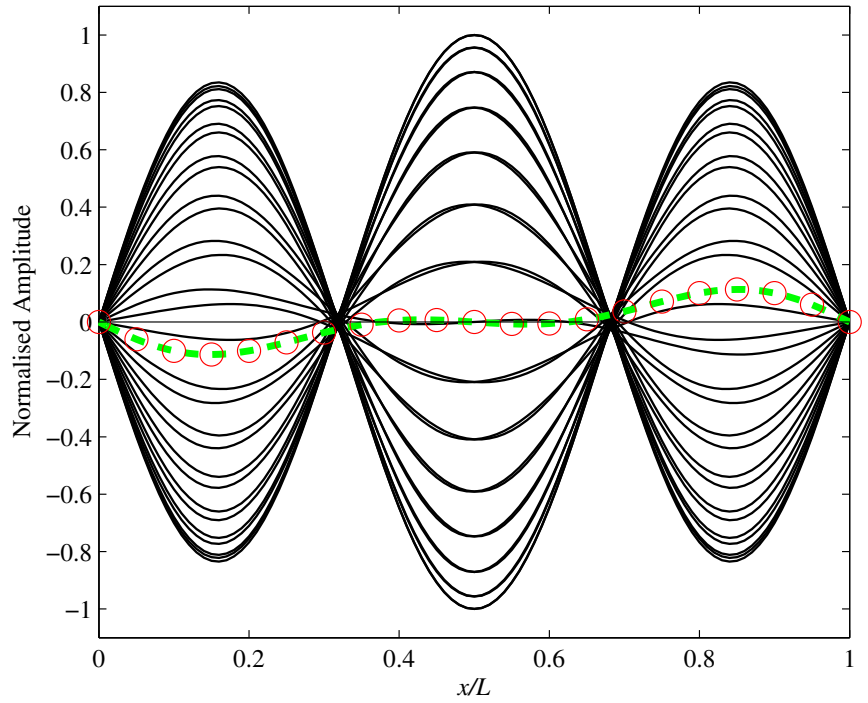

(c)

Figure 6: 


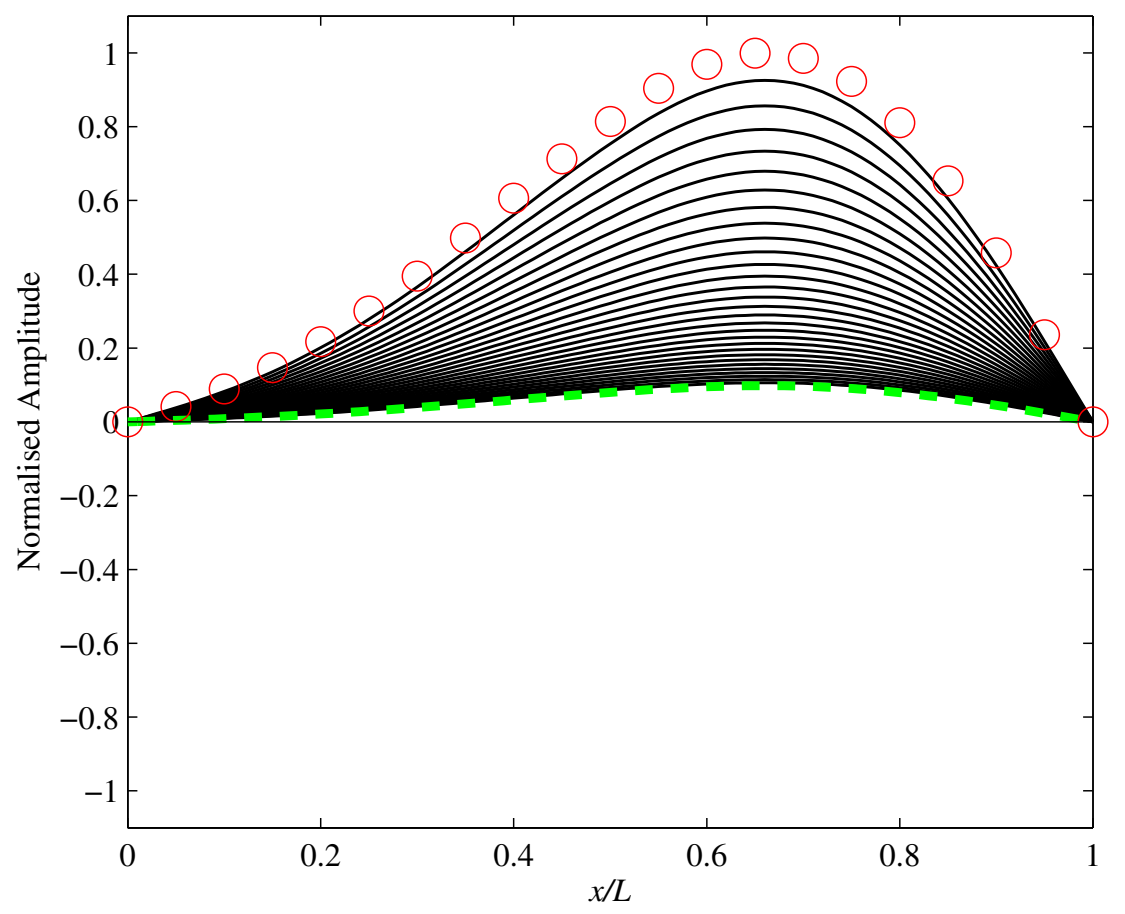

(a)

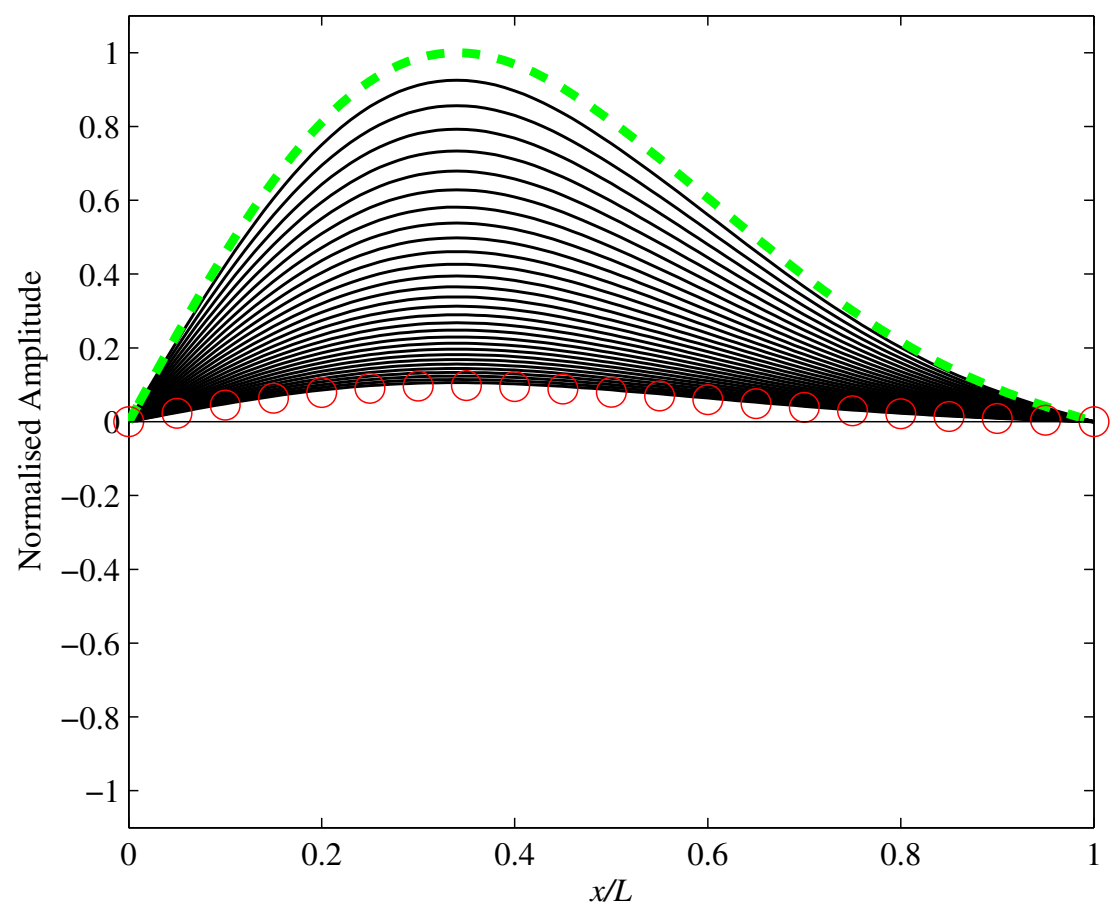

(b)

Figure 7: 


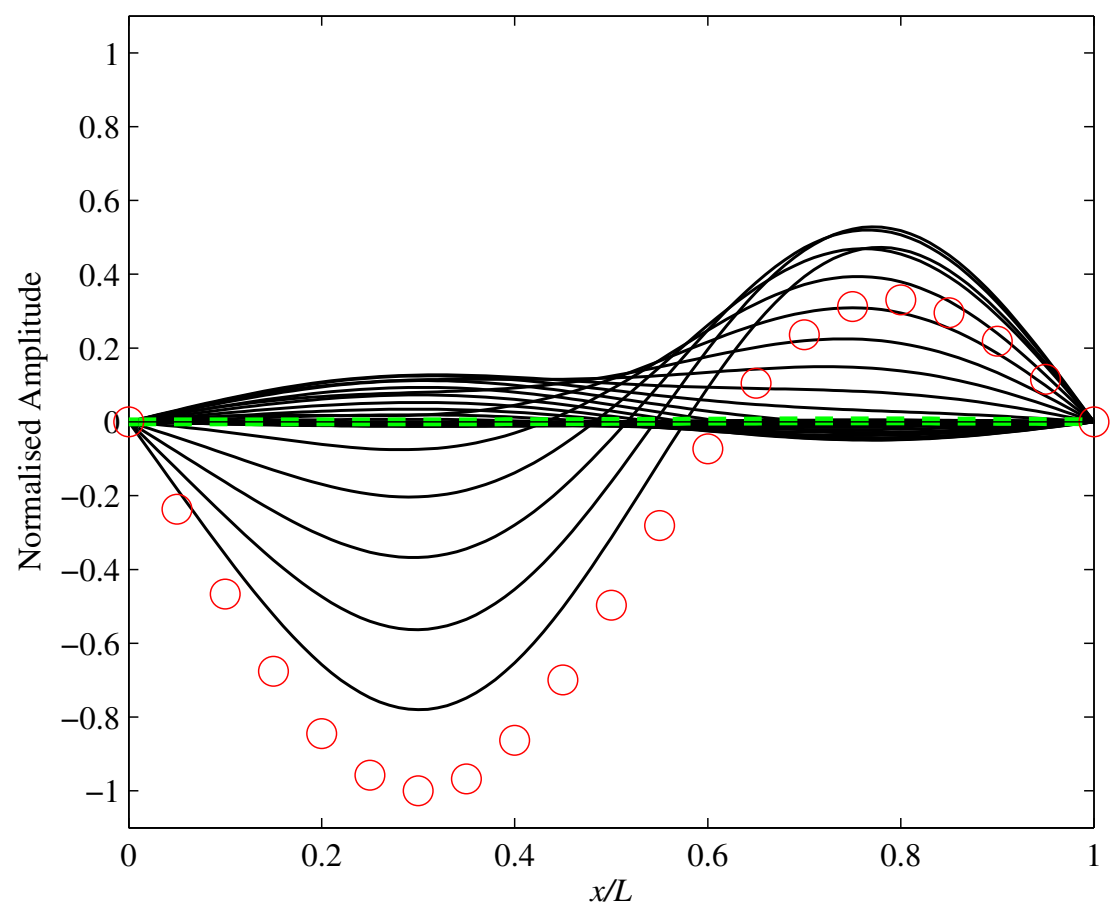

(a)

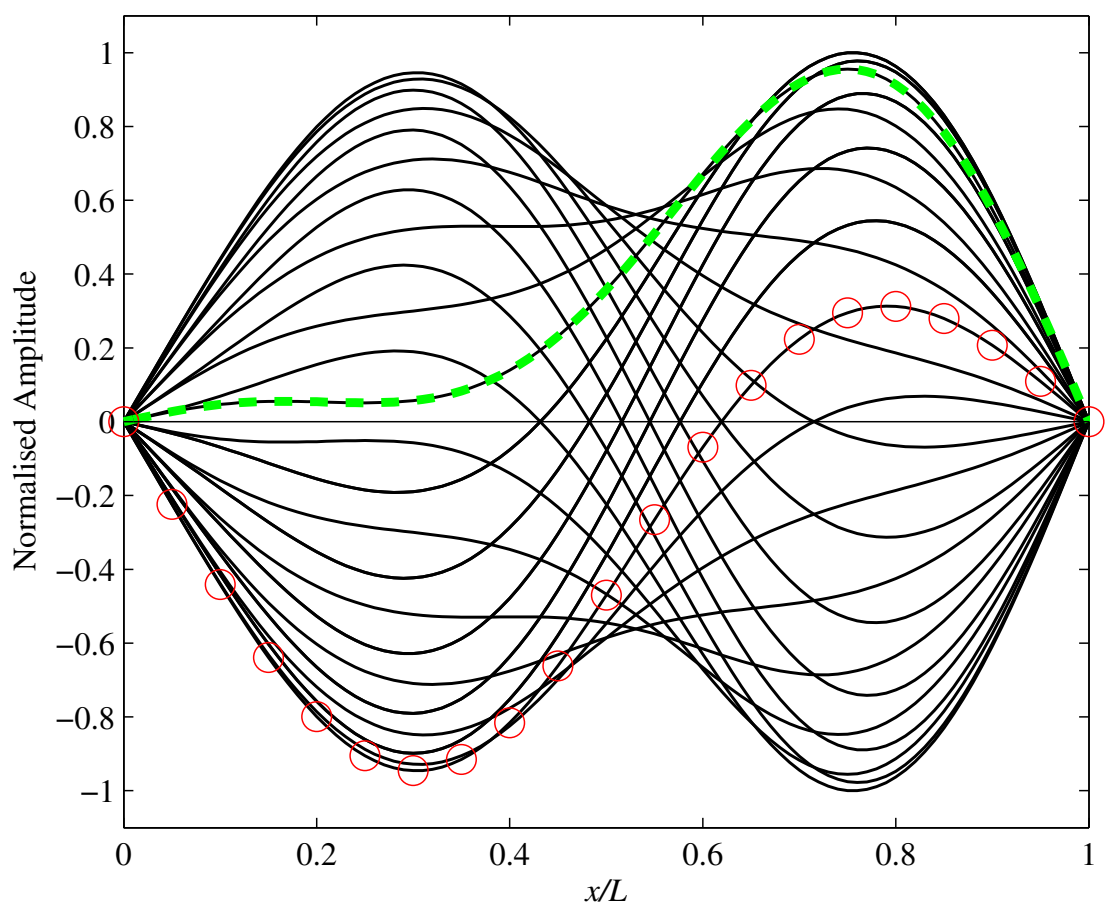

(b)

Figure 8: 


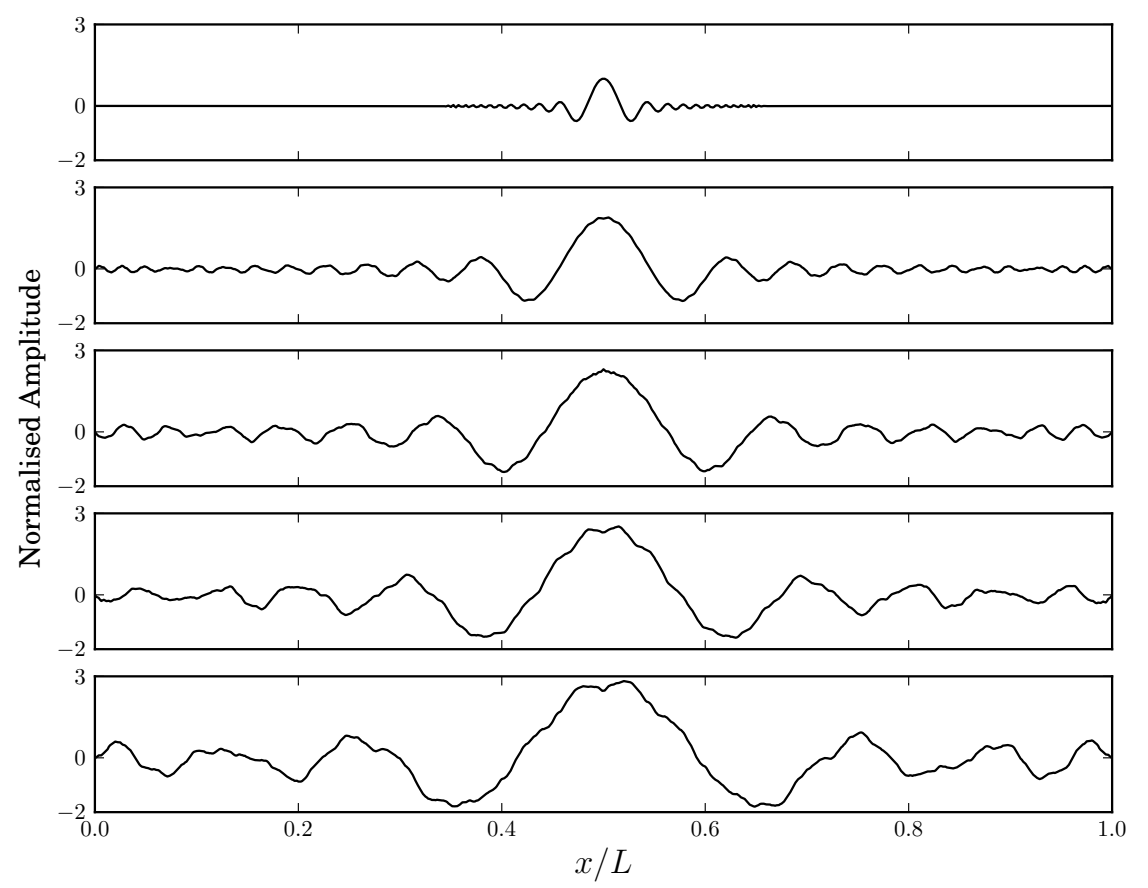

(a)

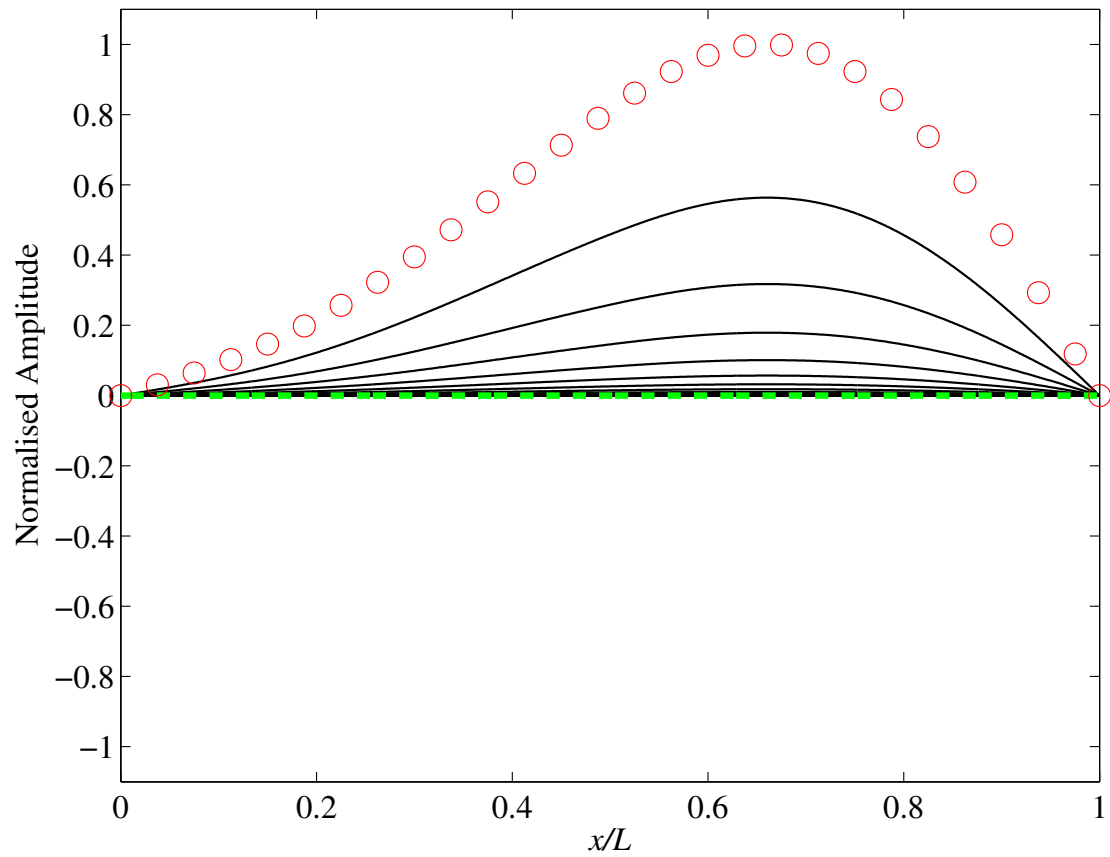

(b)

Figure 9: 


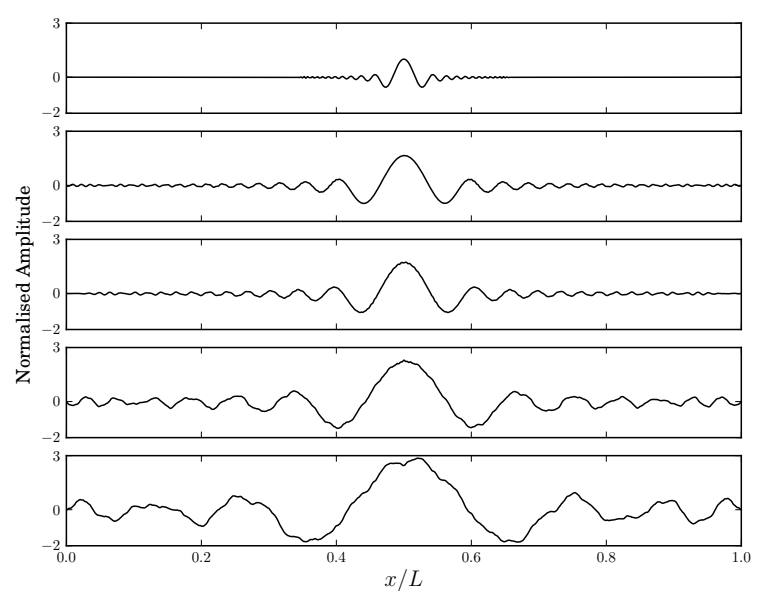

(a)

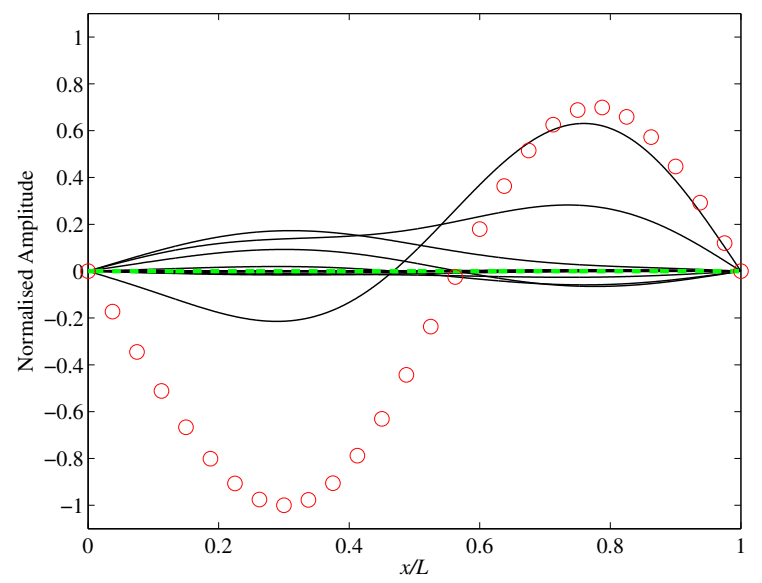

(c)

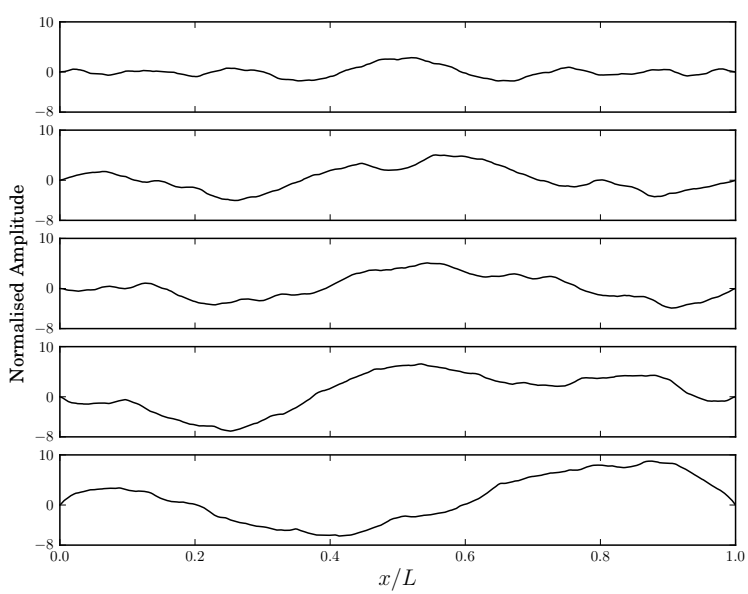

(b)

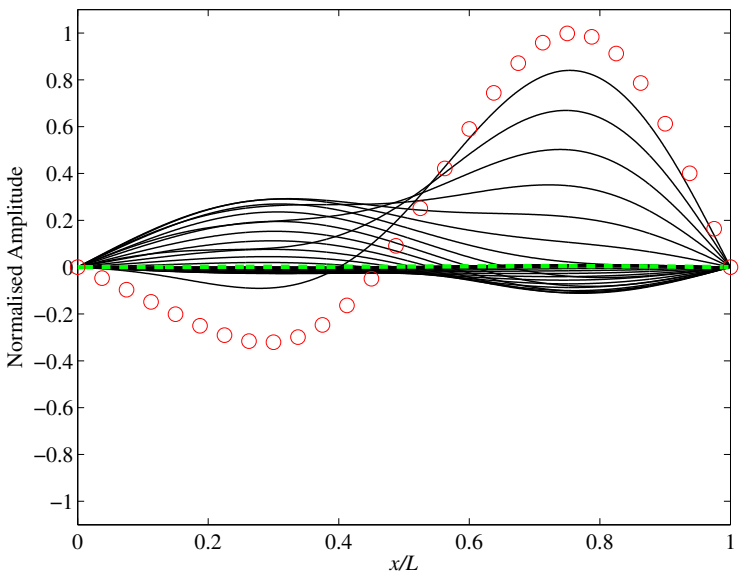

(d)

Figure 10: 


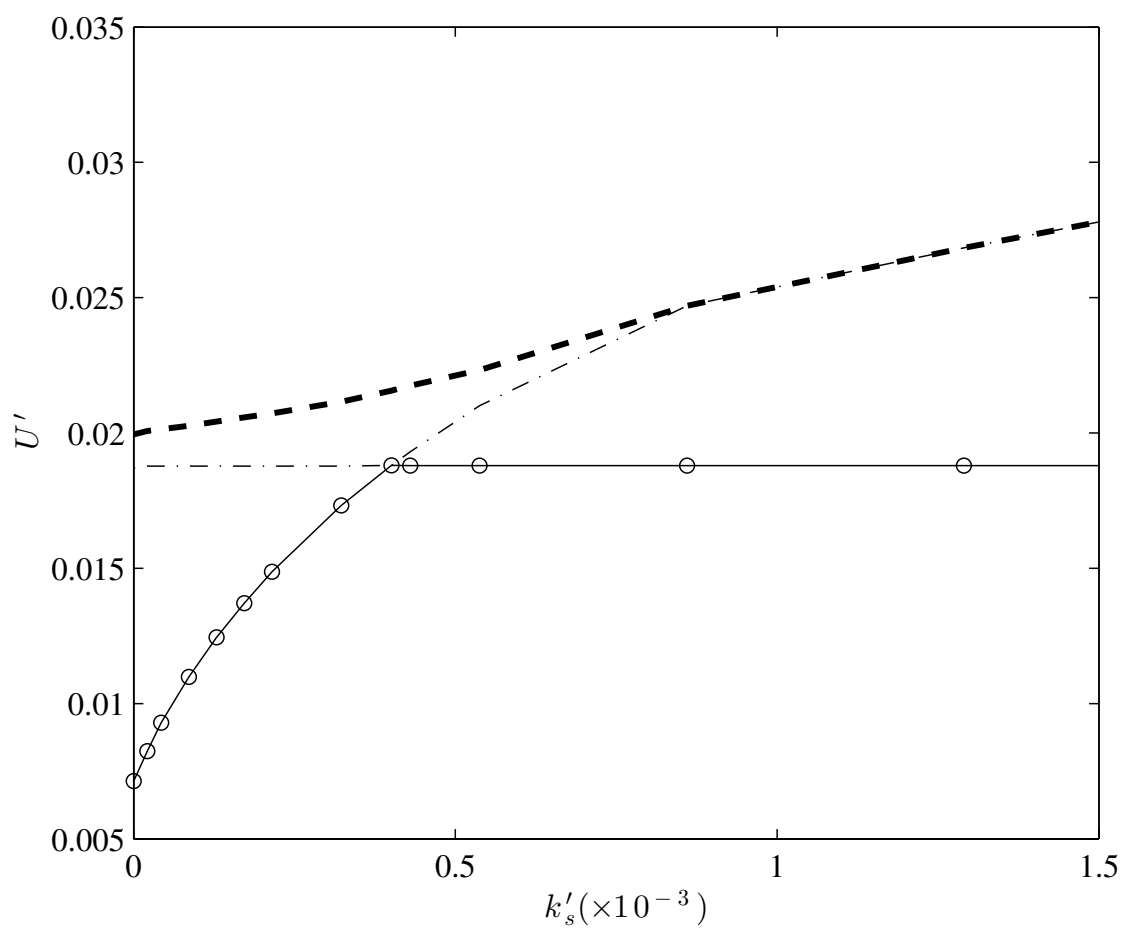

(a)

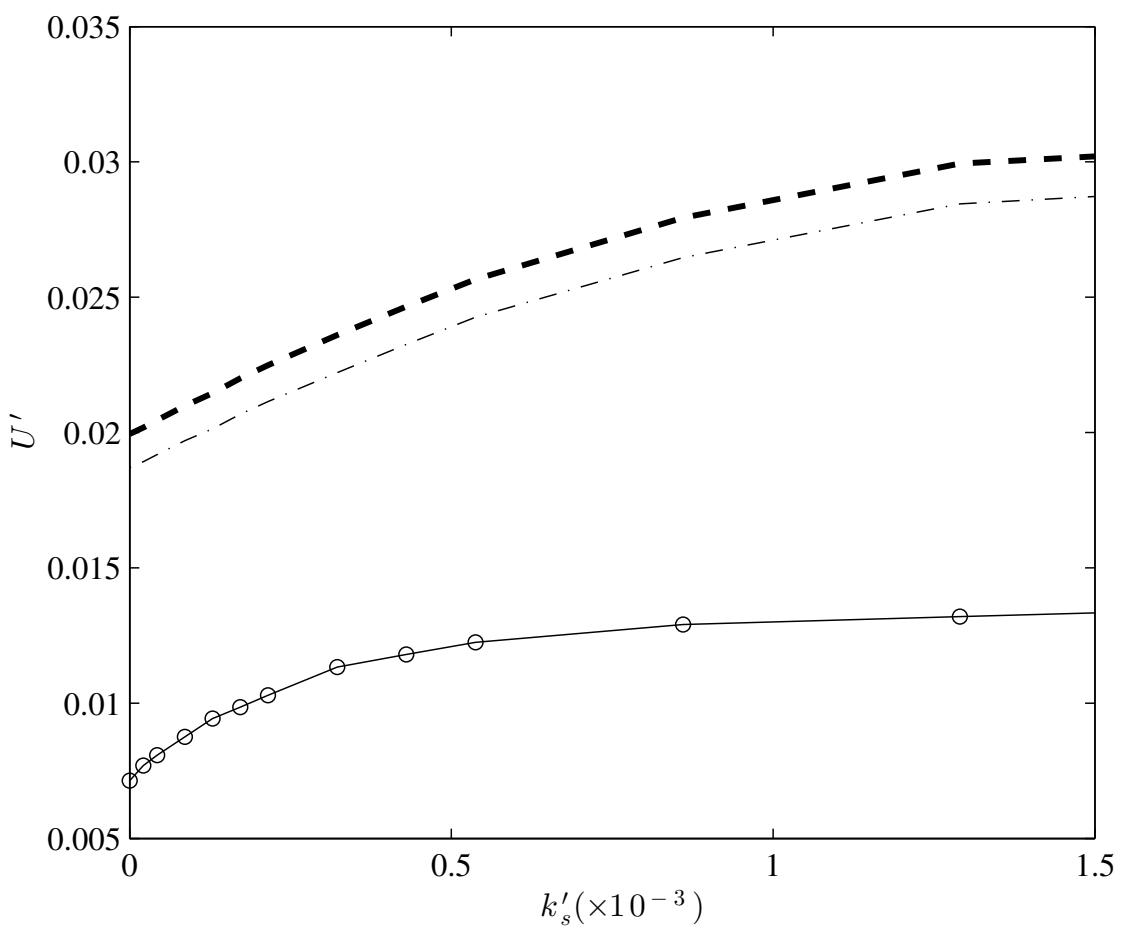

(b)

Figure 11: 


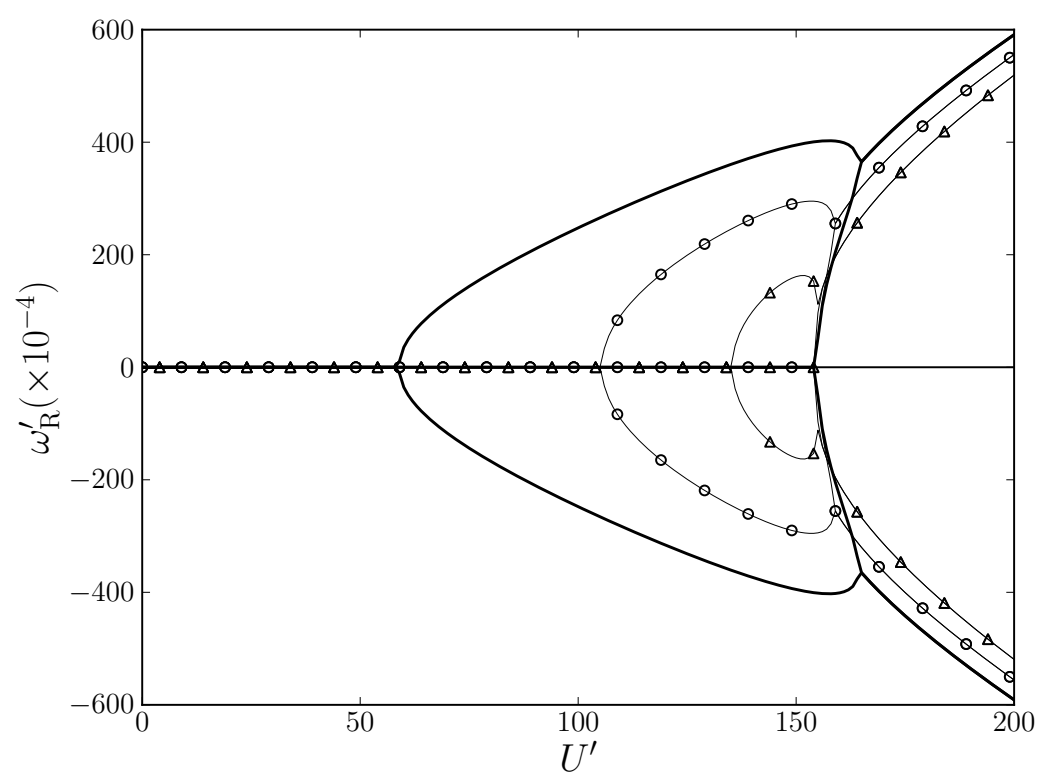

(a)

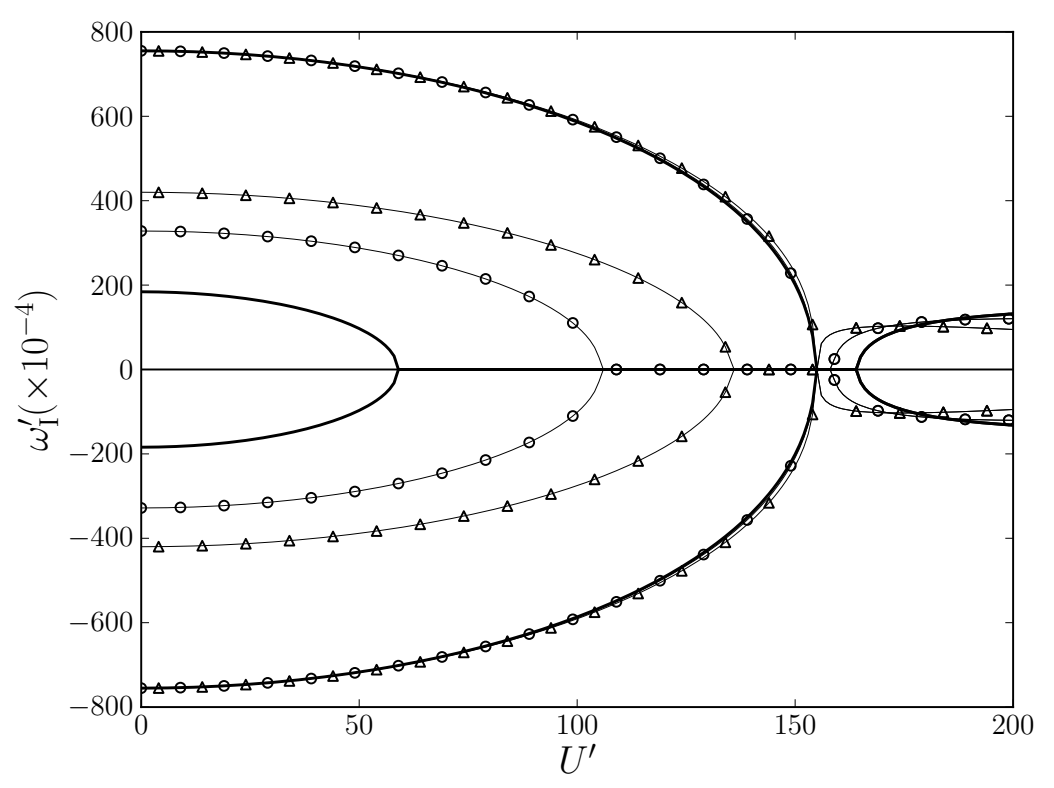

(b)

Figure 12: 


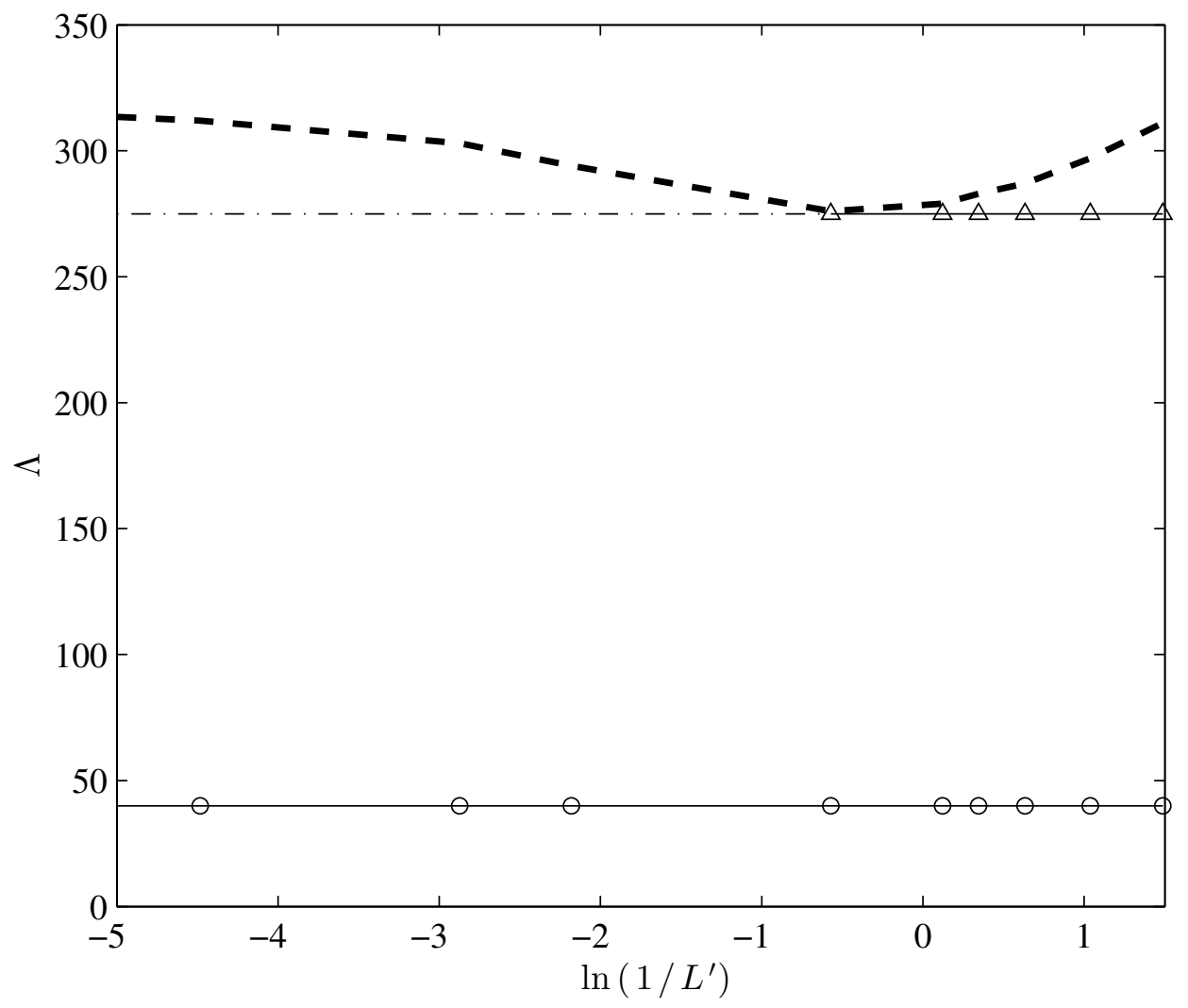

Figure 13: 


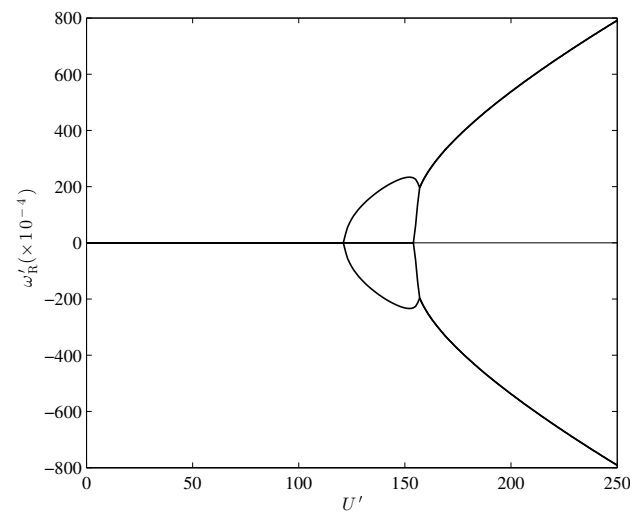

(a)

है

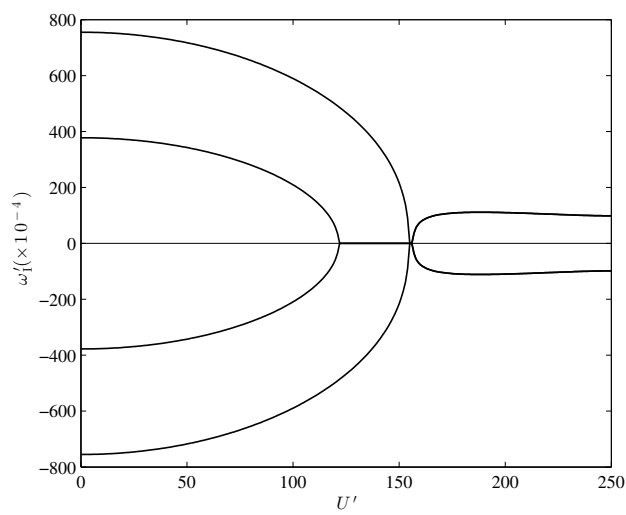

(d)

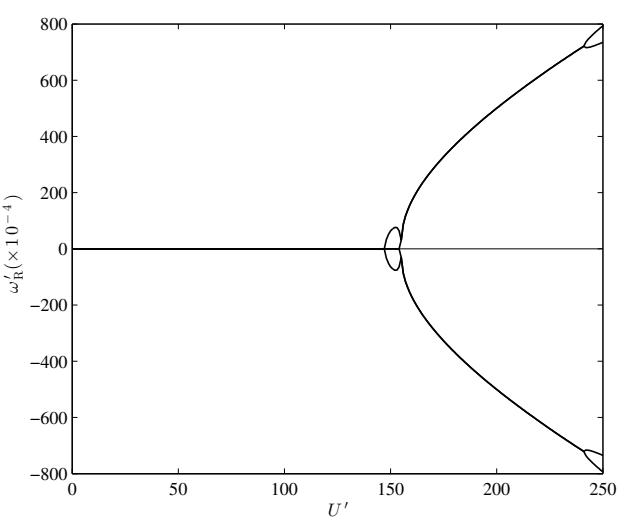

(b)

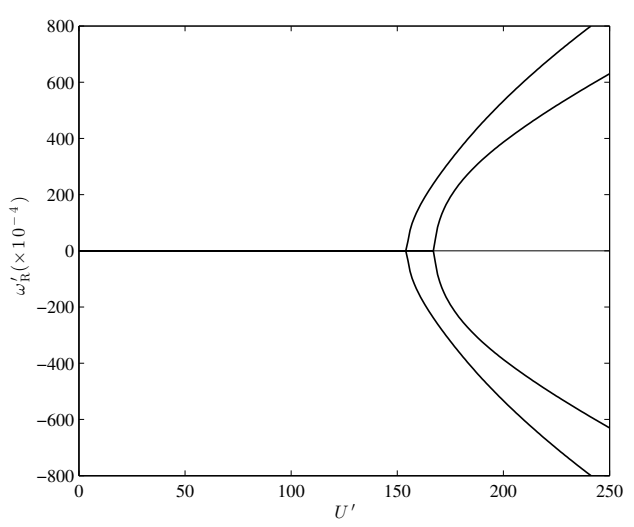

(c)

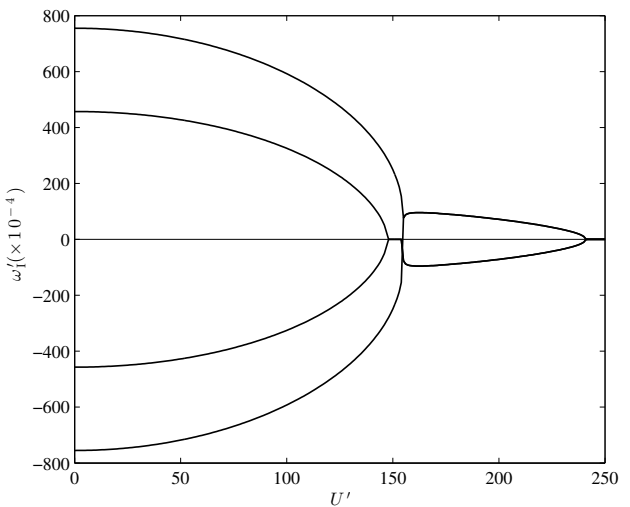

(e)

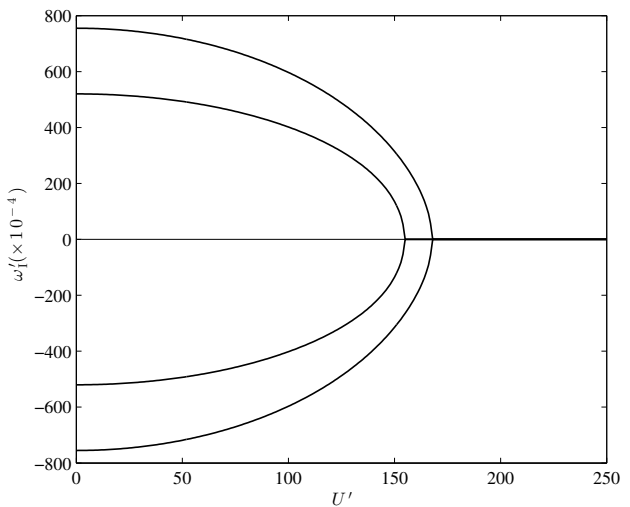

(f)

Figure 14: 


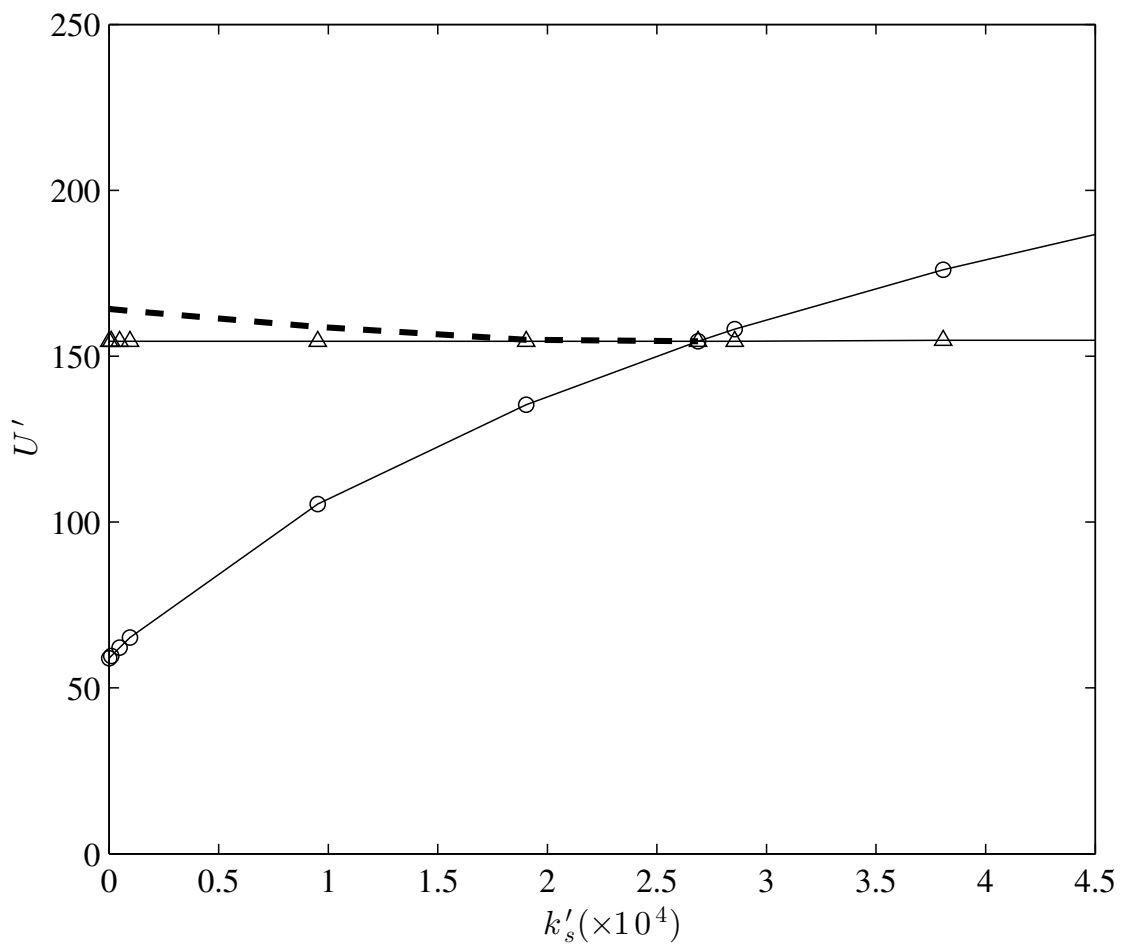

(a)

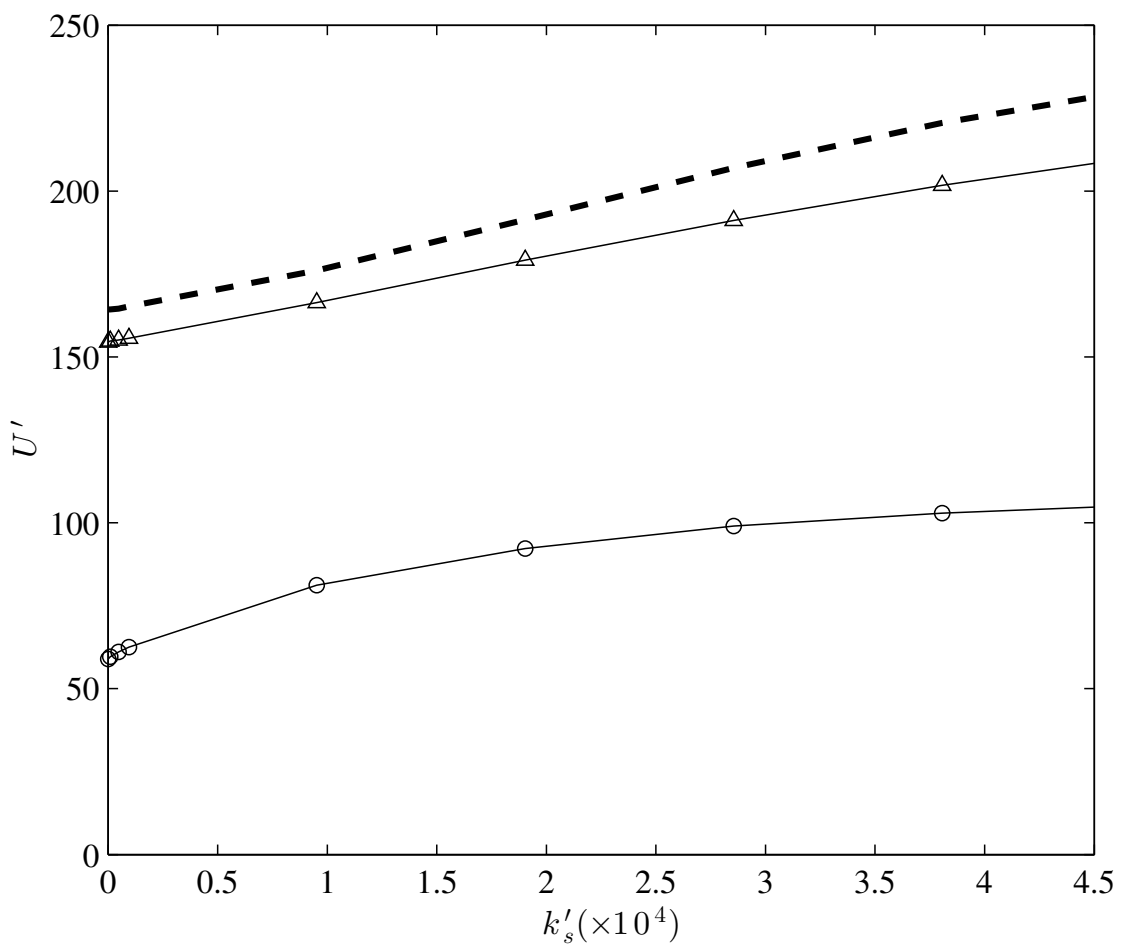

(b)

Figure 15: 


\begin{tabular}{cccc}
\hline \hline \multirow{2}{*}{$\begin{array}{c}\text { Spring } \\
\text { constant }\end{array}$} & \multicolumn{3}{c}{$L / h$} \\
\cline { 2 - 4 }$\left(k_{+}=6 \times 10^{3} \mathrm{~N} / \mathrm{m}^{2}\right)$ & Panel (i) & Panel (ii) & Panel (iii) \\
\hline \multirow{2}{*}{$0 k_{+}$} & $3.7 \mathrm{~m} / \mathrm{s}$ & $7.6 \mathrm{~m} / \mathrm{s}$ & $8.1 \mathrm{~m} / \mathrm{s}$ \\
& $(7.2 \mathrm{knots})$ & $(14.7 \mathrm{knots})$ & $(15.7 \mathrm{knots})$ \\
\hline \multirow{2}{*}{$6 k_{+}$} & $6.5 \mathrm{~m} / \mathrm{s}$ & $9.3 \mathrm{~m} / \mathrm{s}$ & $9.8 \mathrm{~m} / \mathrm{s}$ \\
& $(12.6 \mathrm{knots})$ & $(18.0 \mathrm{knots})$ & $(19.0 \mathrm{knots})$ \\
\hline $15 k_{+}$ & $9.2 \mathrm{~m} / \mathrm{s}$ & $11.4 \mathrm{~m} / \mathrm{s}$ & $11.8 \mathrm{~m} / \mathrm{s}$ \\
& $(17.8 \mathrm{knots})$ & $(22.1 \mathrm{knots})$ & $(22.9 \mathrm{knots})$ \\
\hline \hline
\end{tabular}

Table 1:

\begin{tabular}{|c|c|c|c|}
\hline \multirow{3}{*}{$\begin{array}{c}\text { Spring } \\
\text { constant } \\
\left(k_{+}=6 \times 10^{3} \mathrm{~N} / \mathrm{m}^{2}\right)\end{array}$} & \multicolumn{3}{|c|}{$L$} \\
\hline & $0.6 \mathrm{~m}$ & $1.2 \mathrm{~m}$ & $0.9144 \mathrm{~m}$ \\
\hline & Panel (i) & Panel (ii) & Panel (iii) \\
\hline $0 k_{+}$ & $\begin{array}{c}2.5 \mathrm{~mm} \\
\text { (standard) }\end{array}$ & $\begin{array}{c}8.0 \mathrm{~mm} \\
\text { (standard) }\end{array}$ & $\begin{array}{r}6.35 \mathrm{~mm} \\
\text { (standard) }\end{array}$ \\
\hline $6 k_{+}$ & $\begin{array}{c}1.7 \mathrm{~mm} \\
(31 \%)\end{array}$ & $\begin{array}{c}7.0 \mathrm{~mm} \\
(13 \%)\end{array}$ & $\begin{array}{c}5.6 \mathrm{~mm} \\
(12 \%)\end{array}$ \\
\hline $15 k_{+}$ & $\begin{array}{c}1.4 \mathrm{~mm} \\
(46 \%)\end{array}$ & $\begin{array}{c}6.1 \mathrm{~mm} \\
(24 \%)\end{array}$ & $\begin{array}{c}4.9 \mathrm{~mm} \\
(22 \%)\end{array}$ \\
\hline
\end{tabular}

Table 2:

\begin{tabular}{cccc}
\hline \hline \multirow{2}{*}{$\begin{array}{c}\text { Spring } \\
\text { constant }\end{array}$} & 533 & $L / h$ & \\
\cline { 2 - 4 }$\left(k_{+}=8500 \mathrm{~N} / \mathrm{m}^{2}\right)$ & $(L=2.135 \mathrm{~m}, h=4 \mathrm{~mm})$ & $(L=2.440 \mathrm{~m}, h=6 \mathrm{~mm})$ & $(L=3.66 \mathrm{~m}, h=10 \mathrm{~mm})$ \\
\hline $0 k_{+}$ & $126 \mathrm{~km} / \mathrm{h}$ & $189 \mathrm{~km} / \mathrm{h}$ & $219 \mathrm{~km} / \mathrm{h}$ \\
\hline $0.05 k_{+}$ & $139 \mathrm{~km} / \mathrm{h}$ & $200 \mathrm{~km} / \mathrm{h}$ & $230 \mathrm{~km} / \mathrm{h}$ \\
\hline $0.5 k_{+}$ & $224 \mathrm{~km} / \mathrm{h}$ & $266 \mathrm{~km} / \mathrm{h}$ & $287 \mathrm{~km} / \mathrm{h}$ \\
\hline \hline
\end{tabular}

Table 3: 